\title{
Free Boundary Problems and Transonic Shocks for the Euler Equations in Unbounded Domains
}

\author{
GUI-QIANG CHEN - MIKHAIL FELDMAN
}

\begin{abstract}
We establish the existence and stability of multidimensional transonic shocks (hyperbolic-elliptic shocks), which are not nearly orthogonal to the flow direction, for the Euler equations for steady compressible potential fluids in unbounded domains in $\mathbb{R}^{n}, n \geq 3$. The Euler equations can be written as a second order nonlinear equation of mixed hyperbolic-elliptic type for the velocity potential. The transonic shock problem can be formulated into the following free boundary problem: The free boundary is the location of the multidimensional transonic shock which divides two regions of $C^{2, \alpha}$ flow, and the equation is hyperbolic in the upstream region where the $C^{2, \alpha}$ perturbed flow is supersonic. In this paper, we develop a new approach to deal with such free boundary problems and establish the existence and stability of multidimensional transonic shocks near planes. We first reformulate the free boundary problem into a fixed conormal boundary value problem for a nonlinear elliptic equation of second order in unbounded domains and then develop techniques to solve this elliptic problem. Our results indicate that there exists a solution of the free boundary problem such that the equation is always elliptic in the unbounded downstream region, the uniform velocity state at infinity in the downstream direction is equal to the unperturbed downstream velocity state, and the free boundary is $C^{2, \alpha}$, provided that the hyperbolic phase is close in $C^{2, \alpha}$ to a uniform flow. We further prove that the free boundary is stable under the $C^{2, \alpha}$ steady perturbation of the hyperbolic phase. Moreover, we extend our existence results to the case that the regularity of the steady perturbation is only $C^{1,1}$, and we introduce another simpler approach to deal with the existence and stability problem when the regularity of the steady perturbation is $C^{3, \alpha}$ or higher. We also establish the existence and stability of multidimensional transonic shocks near spheres in $\mathbb{R}^{n}$.
\end{abstract}

Mathematics Subject Classification (2000): 35M10, 35J65, 35R35, 76L05 (primary); 76H05, 35B45, 35B35 (secondary).

\section{1. - Introduction}

We are concerned with the existence and stability of multidimensional steady transonic shocks, which are not nearly orthogonal to the flow direction, in 
inviscid compressible potential flows. The Euler equations for such fluid flows consist of the conservation law of mass and the Bernoulli law for velocity, and can be formulated into the following nonlinear second-order equations of mixed elliptic-hyperbolic type for the velocity potential $\varphi: \Omega \subset \mathbb{R}^{n} \rightarrow \mathbb{R}$ :

$$
\operatorname{div}\left(\rho\left(|D \varphi|^{2}\right) D \varphi\right)=0,
$$

where the density $\rho\left(q^{2}\right)$ is

$$
\rho\left(q^{2}\right)=\left(1-\frac{\gamma-1}{2} q^{2}\right)^{\frac{1}{\gamma-1}}
$$

for the adiabatic exponent $\gamma>1$. The second-order nonlinear equation (1.1) is elliptic at $D \varphi$ with $|D \varphi|=q$ if

$$
\rho\left(q^{2}\right)+2 q^{2} \rho^{\prime}\left(q^{2}\right)>0 ;
$$

and is hyperbolic if

$$
\rho\left(q^{2}\right)+2 q^{2} \rho^{\prime}\left(q^{2}\right)<0 .
$$

Some efforts were made in solving the nonlinear equation (1.1) of mixed elliptichyperbolic type in [4], [8], [9], [12], [15], [21], [26], [30], [32], [33], [34], and the references cited therein. A similar problem was considered in [5] for the two-dimensional transonic small-disturbance (TSD) model. In [6], we developed a nonlinear approach by combining an iteration scheme with a fixed point technique to establish the existence and stability of multidimensional transonic shocks that are nearly orthogonal to the flow direction. In Sections 3-4, we develop a new, different approach to deal with other difficulties for general multidimensional transonic shock problems, especially including the essential non-orthogonality of transonic shocks to the flow direction; such situations arise in several important physical problems.

In this paper, we first focus on multidimensional transonic shocks near a plane in $\mathbb{R}^{n}, n \geq 3$. Such a transonic shock problem can be formulated into the corresponding free boundary problem: The free boundary is the location of the multidimensional transonic shock which divides two regions of $C^{2, \alpha}$ flow in $\mathbb{R}^{n}$, and the equation is hyperbolic in the upstream region where the $C^{2, \alpha}$ perturbed flow is supersonic. One of the main ingredients in our new approach is to employ a partial hodograph transform to reduce the free boundary problem into a conormal boundary value problem for the corresponding nonlinear elliptic equation of divergence form in the half space. In order to solve the conormal boundary value problem in the unbounded domain, our strategy is to first construct solutions in a series of half balls with radius $R$, then make uniform estimates in $R$, and finally send $R \rightarrow \infty$. To achieve this requires delicate apriori estimates. We first obtain a uniform bound in a weighted $L^{\infty}$-norm by employing a comparison principle and identifying a global function with the 
same decay rate as the fundamental solution of the elliptic equation with constant coefficients which controls the solutions. Then, by scaling arguments, we obtain the uniform estimates in a weighted Hölder norm for the solutions. Thus we obtain the existence of a solution in the half space and the algebraic rate of decay of this solution at infinity. For such decaying solutions in the half space, a comparison principle holds, which implies the uniqueness for the conormal problem. Finally, by the gradient estimate, we show that the limit function is a solution of the multidimensional transonic shock problem, and the existence result can be extended to the case that the regularity of the steady perturbation is only $C^{1,1}$ in Section 4 . We further prove that the multidimensional transonic shock solution is stable with respect to the $C^{2, \alpha}$ supersonic perturbation in Section 5, in which we also introduce another simpler approach to deal with the existence and stability problem when the regularity of the steady perturbation is $C^{3, \alpha}$ or higher.

In Section 6, we extend the approach by using the partial hodograph transform in the radial direction in the polar coordinates to establish the existence and stability of multidimensional transonic shocks near spheres in $\mathbb{R}^{n}, n \geq 3$.

We remark that the case $n=2$ exhibits special features, different from the case $n \geq 3$, and requires different techniques to obtain the uniform estimates of solutions in the weighted Hölder norms in the increasing domains, which will be a part of the content of our forthcoming paper.

ACKNOWLEDGMENTS. Gui-Qiang Chen's research was supported in part by the National Science Foundation under Grants DMS-0244473, DMS-0204225, DMS-0204455, and INT-9987378. Mikhail Feldman's research was supported in part by the National Science Foundation under Grants DMS-0200644 and DMS-0074037. The main part of this paper was completed when the first author visited the Isaac Newton Institute for Mathematical Sciences, University of Cambridge, UK, March-June, 2003, and when the second author visited the Centre for Mathematics and its Applications, Australian National University, Canberra, Australia, June 2002.

\section{2. - Multidimensional transonic shocks in the whole space}

In this section, we first set up the problems of multidimensional transonic shocks near a plane in the whole space $\mathbb{R}^{n}$ and present the main theorems of this paper.

A function $\varphi \in W^{1, \infty}(\Omega)$ is a weak solution of (1.1) in an unbounded domain $\Omega$ if

(i) $|D \varphi(x)| \leq \sqrt{2 /(\gamma-1)}$ a.e.

(ii) For any $w \in C_{0}^{\infty}(\Omega)$,

$$
\int_{\Omega} \rho\left(|D \varphi|^{2}\right) D \varphi \cdot D w d x=0 .
$$


We are interested in weak solutions with shocks. Let $\Omega^{+}$and $\Omega^{-}$be open subsets of $\Omega$ such that

$$
\Omega^{+} \cap \Omega^{-}=\emptyset, \quad \overline{\Omega^{+}} \cup \overline{\Omega^{-}}=\bar{\Omega},
$$

and $S=\partial \Omega^{+} \cap \Omega$. Let $\varphi \in W^{1, \infty}(\Omega)$ satisfy $\varphi \in C^{2}\left(\Omega^{ \pm}\right) \cap C^{1}\left(\overline{\Omega^{ \pm}}\right)$so that $D \varphi$ experiences a jump across $S$ that is an $(n-1)$-dimensional smooth surface. Then $\varphi$ is a weak solution of (1.1) if and only if $|D \varphi| \leq \sqrt{2 /(\gamma-1)}$ in $\Omega^{ \pm}$ and the following two conditions hold on $S$ : First,

$$
\varphi^{+}=\varphi^{-} \quad \text { on } S,
$$

where $\varphi^{ \pm}$denotes $\varphi$ in $\Omega^{ \pm}$, respectively; Second, the Rankine-Hugoniot jump condition on $S$ :

$$
\left[\rho\left(|D \varphi|^{2}\right) D \varphi \cdot v\right]_{S}=0,
$$

where $v$ is the unit normal to $S$ from $\Omega^{-}$to $\Omega^{+}$, and the bracket denotes the difference between the values of the function along $S$ on the $\Omega^{ \pm}$sides of $S$, respectively. We can also write (2.3) as

$$
\rho\left(\left|D \varphi^{+}\right|^{2}\right) \varphi_{v}^{+}=\rho\left(\left|D \varphi^{-}\right|^{2}\right) \varphi_{v}^{-} \quad \text { on } \quad S,
$$

where $\varphi_{v}^{ \pm}=D \varphi^{ \pm} \cdot v$ are the normal derivatives on the $\Omega^{ \pm}$sides, respectively.

The function

$$
\Phi(p):=\left(1-\frac{\gamma-1}{2} p^{2}\right)^{\frac{1}{\gamma-1}} p
$$

defined for $p \in(0, \sqrt{2 /(\gamma-1)})$, satisfies

$$
\begin{aligned}
& \lim _{p \rightarrow 0+} \Phi(p)=\lim _{p \rightarrow \sqrt{2 /(\gamma-1)}-} \Phi(p)=0, \quad \Phi(p)>0 \text { for } p \in(0, \sqrt{2 /(\gamma-1)}) \\
& 0<\Phi^{\prime}(p)<1 \text { on }\left(0, c_{*}\right), \quad \Phi^{\prime}(p)<0 \text { on }\left(c_{*}, \sqrt{2 /(\gamma-1)}\right) \\
& \Phi^{\prime \prime}(p)<0 \text { on }\left(0, c_{*}\right]
\end{aligned}
$$

where

$$
c_{*}:=\sqrt{2 /(\gamma+1)}
$$

is the sonic speed.

Suppose that $\varphi(x)$ is a solution satisfying

$$
|D \varphi(x)|<c_{*} \quad \text { in } \Omega^{+}, \quad|D \varphi(x)|>c_{*} \quad \text { in } \Omega^{-},
$$


and

$$
D \varphi^{ \pm} \cdot v>0 \quad \text { on } S
$$

besides (2.2) and (2.3). Then $\varphi(x)$ is a transonic shock solution with transonic shock $S$ dividing subsonic region $\Omega^{+}$and supersonic region $\Omega^{-}$and satisfying the physical entropy condition (see Courant-Friedrichs [10]; also see Dafermos [11] and Lax [20]):

$$
\rho\left(\left|D \varphi^{-}\right|^{2}\right)<\rho\left(\left|D \varphi^{+}\right|^{2}\right) \quad \text { along } \quad S,
$$

which implies, by (2.11), that the density increases in the flow direction. Note that equation (1.1) is elliptic in the subsonic region and is hyperbolic in the supersonic region.

Let $\left(x^{\prime}, x_{n}\right)$ be the coordinates in $\mathbb{R}^{n}$, where $x^{\prime}=\left(x_{1}, \ldots, x_{n-1}\right) \in \mathbb{R}^{n-1}$ and $x_{n} \in \mathbb{R}$. Fix $V_{0} \in \mathbb{R}^{n}$, and let

$$
\varphi_{0}(x):=V_{0} \cdot x, \quad x \in \mathbb{R}^{n} .
$$

If $\left|V_{0}\right| \in\left(0, c_{*}\right)$ (resp. $\left|V_{0}\right| \in\left(c_{*}, \sqrt{2 /(\gamma-1)}\right)$ ), then $\varphi_{0}(x)$ is a subsonic (resp. supersonic) solution in $\mathbb{R}^{n}$, and $V_{0}=D \varphi_{0}$ is its velocity.

Let $V_{0}^{\prime} \in \mathbb{R}^{n-1}$ and $q_{0}^{+}>0$ be such that the vector $V_{0}^{+}:=\left(V_{0}^{\prime}, q_{0}^{+}\right)$satisfies $\left|V_{0}^{+}\right|<c_{*}$. Then, using the properties of function (2.5), we conclude from (2.6)-(2.9) that there exists a unique $q_{0}^{-}>q_{0}^{+}$such that

$$
\left(1-\frac{\gamma-1}{2}\left(\left|V_{0}^{\prime}\right|^{2}+\left|q_{0}^{+}\right|^{2}\right)\right)^{\frac{1}{\gamma-1}} q_{0}^{+}=\left(1-\frac{\gamma-1}{2}\left(\left|V_{0}^{\prime}\right|^{2}+\left|q_{0}^{-}\right|^{2}\right)\right)^{\frac{1}{\gamma-1}} q_{0}^{-}
$$

Moreover, $\left|\left(V_{0}^{\prime}, q_{0}^{-}\right)\right|>c_{*}$. By denoting $V_{0}^{-}:=\left(V_{0}^{\prime}, q_{0}^{-}\right)$and defining functions $\varphi_{0}^{ \pm}(x):=V_{0}^{ \pm} \cdot x$ on $\mathbb{R}^{n}$, then $\varphi_{0}^{+}$(resp. $\varphi_{0}^{-}$) is a subsonic (resp. supersonic) solution. Furthermore, from (2.4) and (2.13), the function

$$
\varphi_{0}(x):=\min \left(\varphi_{0}^{+}(x), \varphi_{0}^{-}(x)\right)= \begin{cases}V_{0}^{-} \cdot x, & x \in \Omega_{0}^{-}:=\left\{x \in \mathbb{R}^{n}: x_{n}<0\right\}, \\ V_{0}^{+} \cdot x, & x \in \Omega_{0}^{+}:=\left\{x \in \mathbb{R}^{n}: x_{n}>0\right\}\end{cases}
$$

is a plane transonic shock solution in $\mathbb{R}^{n}, \Omega_{0}^{+}$and $\Omega_{0}^{-}$are respectively its subsonic and supersonic regions, and $S=\left\{x_{n}=0\right\}$ is a transonic shock. Note that, if $V_{0}^{\prime}=0$, then the velocities $V_{0}^{ \pm}$are orthogonal to the shock $S$ and, if $V_{0}^{\prime} \neq 0$, then the velocities are not orthogonal to $S$.

The multidimensional transonic shock problem near $\varphi_{0}(x)$ with $V_{0}^{\prime}=0$ has been handled in Chen-Feldman [6], [7]. In this paper, we develop a new approach to handle with the case $V_{0}^{\prime} \neq 0$ in the whole space $\mathbb{R}^{n}$. We first study perturbations of the uniform transonic shock solution (2.14) in the whole space $\mathbb{R}^{n}, n \geq 3$, in Sections 3-5. In order to state our problem, we first introduce weighted Hölder seminorms and norms on unbounded domains. Note that later 
we consider our fixed boundary value problems on the subsonic region $\Omega^{+}$, which is expected to be close to the half-space $\Omega_{0}^{+}=\left\{x_{n}>0\right\}$.

Let $\mathcal{D}=\left\{x_{n}>f\left(x^{\prime}\right)\right\}$, where $f\left(x^{\prime}\right)$ is a Lipschitz function. For $x=$ $\left(x^{\prime}, x_{n}\right) \in \mathcal{D}$, let $\hat{\delta}_{x}=1+|x|$ and, for $x, y \in \mathcal{D}$, let $\hat{\delta}_{x, y}=1+\min \left(\delta_{x}, \delta_{y}\right)$. Let $\theta \in \mathbb{R}, \alpha \in(0,1)$, and $k$ a nonnegative integer. We define

$$
\begin{aligned}
{[[u]]_{k ; 0 ; \mathcal{D}}^{(\theta)} } & =\sup _{x \in \mathcal{D}}\left(\hat{\delta}_{x}^{k+\theta}\left|D^{k} u(x)\right|\right), \\
{[[u]]_{k ; \alpha ; \mathcal{D}}^{(\theta)} } & =\sup _{x, y \in \mathcal{D}, x \neq y}\left(\hat{\delta}_{x, y}^{k+\alpha+\theta} \frac{\left|D^{k} u(x)-D^{k} u(y)\right|}{|x-y|^{\alpha}}\right), \\
\|u\|_{k ; 0 ; \mathcal{D}}^{(\theta)} & =\sum_{j=0}^{k}[[u]]_{j ; 0 ; \mathcal{D}}^{(\theta)}, \quad\|u\|_{k ; \alpha ; \mathcal{D}}^{(\theta)}=\|u\|_{k ; 0 ; \mathcal{D}}^{(\theta)}+[[u]]_{k ; \alpha ; \mathcal{D}}^{(\theta)} .
\end{aligned}
$$

We study the existence and stability of multidimensional transonic shocks near the plane transonic shock (2.14) under small perturbations of the supersonic flow. It suffices to prescribe the perturbed supersonic flow only near the unperturbed shock surface $S_{0}=\left\{x_{n}=0\right\}$. Thus, we introduce a domain $\Omega_{1}:=\mathbb{R}^{n-1} \times(-1,1)$ and focus our discussion on the domain $\Omega:=\mathbb{R}^{n-1} \times(-1, \infty)$.

Problem A. Given a supersonic solution $\varphi^{-}(x)$ of $(1.1)$ in $\Omega_{1}$ satisfying that, for some $\alpha>0$,

$$
\left\|\varphi^{-}-\varphi_{0}^{-}\right\|_{2, \alpha, \Omega_{1}}^{(n-1)} \leq \sigma
$$

with $\sigma>0$ small, find a transonic shock solution $\varphi(x)$ in $\Omega$ such that $\Omega^{-} \subset \Omega_{1}$ and $\varphi(x)=\varphi^{-}(x)$ in $\Omega^{-}$, where $\Omega^{-}:=\Omega \backslash \Omega^{+}$and $\Omega^{+}:=\{x \in \Omega:|D \varphi(x)|<$ $\left.c_{*}\right\}$, and

$$
\begin{aligned}
& \varphi=\varphi^{-}, \quad \partial_{x_{n}} \varphi=\partial_{x_{n}} \varphi^{-} \quad \text { on }\left\{x_{n}=-1\right\}, \\
& \lim _{R \rightarrow \infty}\left\|\varphi-\varphi_{0}^{+}\right\|_{C^{1}\left(\Omega^{+} \backslash B_{R}(0)\right)}=0 .
\end{aligned}
$$

REMARK 2.1. Condition (2.17) determines that the solution is supersonic upstream, while condition (2.18) determines, in particular, that the uniform velocity state at infinity in the downstream direction is equal to the unperturbed downstream velocity state. The additional requirement in (2.18) that $\varphi \rightarrow \varphi_{0}^{+}$ at infinity within $\Omega^{+}$fixes the position of shock at infinity. This allows to determine the solution of Problem A uniquely.

REMARK 2.2. Note that our assumptions imply that the perturbation is not only small in $C^{2, \alpha}$, but also "localized", i.e., has an appropriate algebraic decay at infinity.

One of our main results of this paper is the following. 
THEOREM 2.1. Let $n \geq 3$. Let $\left|\left(V_{0}^{\prime}, q_{0}^{+}\right)\right| \in\left(0, c_{*}\right)$ and $\left|\left(V_{0}^{\prime}, q_{0}^{-}\right)\right| \in\left(c_{*}, \sqrt{2 /(\gamma-1)}\right)$ satisfy (2.13), and let $\varphi_{0}(x)$ be the transonic shock solution (2.14). Then there exist positive constants $\sigma_{0}, C_{1}$, and $C_{2}$ depending only on $n, \gamma, \alpha,\left|V_{0}^{\prime}\right|$, and $q_{0}^{+}$such that, for every $\sigma \leq \sigma_{0}$ and any supersonic solution $\varphi^{-}(x)$ of (1.1) satisfying the conditions stated in Problem A, there exists a unique solution $\varphi(x)$ of Problem A satisfying

$$
\left\|\varphi-\varphi_{0}^{+}\right\|_{2, \alpha, \Omega^{+}}^{(n-2)} \leq C_{1} \sigma
$$

with $\Omega^{+}$defined in Problem A. In addition,

$$
\Omega^{+}=\left\{x_{n}>f\left(x^{\prime}\right)\right\},
$$

where $f: \mathbb{R}^{n-1} \rightarrow \mathbb{R}$ satisfies

$$
\|f\|_{2, \alpha, \mathbb{R}^{n-1}}^{(n-2)} \leq C_{2} \sigma,
$$

that is, the shock surface $S=\left\{\left(x^{\prime}, x_{n}\right): x_{n}=f\left(x^{\prime}\right), x^{\prime} \in \mathbb{R}^{n-1}\right\}$ is in $C^{2, \alpha}$ and converges at infinity, with an appropriate algebraic rate, to the hyperplane $S_{0}=\left\{x_{n}=0\right\}$.

This existence result can be extended to the case that the regularity of the steady perturbation $\varphi^{-}$is only $C^{1,1}$, that is, (2.16) can be replaced by

$$
\left\|\varphi^{-}-\varphi_{0}^{-}\right\|_{1,1, \Omega_{1}}^{(n-1)} \leq \sigma .
$$

See Remark 4.1. Furthermore, we have the following stability theorem.

THEOREM 2.2. Let $n \geq 3$. There exist a nonnegative nondecreasing function $\Psi \in C([0, \infty))$ satisfying $\Psi(0)=0$ and a constant $\sigma_{0}$ depending only on $n, \gamma, \alpha$, $\left|V_{0}^{\prime}\right|$, and $q_{0}^{+}$such that, if $\sigma<\sigma_{0}$ and smooth supersonic solutions $\varphi^{-}(x)$ and $\hat{\varphi}^{-}(x)$ of (1.1) satisfy (2.16), then the unique solutions $\varphi(x)$ and $\hat{\varphi}(x)$ of Problem A for $\varphi^{-}(x)$ and $\hat{\varphi}^{-}(x)$, respectively, satisfy

$$
\left\|f_{\varphi}-f_{\hat{\varphi}}\right\|_{2, \alpha, \mathbb{R}^{n-1}}^{(n-2)} \leq \Psi\left(\left\|\varphi^{-}-\hat{\varphi}^{-}\right\|_{2, \alpha, \Omega_{1}}^{(n-1)}\right),
$$

where $f_{\varphi}\left(x^{\prime}\right)$ and $f_{\hat{\varphi}}\left(x^{\prime}\right)$ are the free boundary functions (2.20) of $\varphi(x)$ and $\hat{\varphi}(x)$, respectively.

The proof of these theorems is obtained first by reducing Problem A to a free boundary problem for a nonlinear, uniformly elliptic equation and then by developing partial hodograph transform techniques to solve the free boundary problem.

When the regularity of the steady perturbation is $C^{3, \alpha}$ or higher, that is,

$$
\left\|\varphi^{-}-\varphi_{0}^{-}\right\|_{3, \alpha, \Omega_{1}}^{(n-1)} \leq \sigma,
$$

we introduce another approach to obtain a stronger stability result. See Remark 5.1. 


\section{3. - Free boundary problems and a partial hodograph transform}

In this section, we first extend $\varphi^{-}$to the whole space $\mathbb{R}^{n}$, then formulate the transonic shock problems into free boundary problems, and finally reformulate the free boundary problems into fixed conormal boundary value problems for a nonlinear elliptic equation.

\section{1. - Extension of $\varphi^{-}$to the whole space $\mathbb{R}^{n}$}

Since $\varphi^{-}$satisfies $(2.16)$ in the domain $\Omega_{1}:=\mathbb{R}^{n-1} \times(-1,1)$, then we use a standard extension procedure to extend $\varphi^{-}$to $\mathbb{R}^{n}$ so that the extension (still denoted) $\varphi^{-}$is in $C^{2, \alpha}\left(\mathbb{R}^{n}\right)$ and satisfies

$$
\begin{aligned}
& \left\|\varphi^{-}-\varphi_{0}^{-}\right\|_{2, \alpha, \mathbb{R}^{n}}^{(n-1)} \leq C(n, \alpha) \sigma, \\
& \operatorname{supp}\left(\varphi^{-}-\varphi_{0}^{-}\right) \subset \mathbb{R}^{n-1} \times(-2,2) .
\end{aligned}
$$

Consider the function

$$
g=\operatorname{div}\left(\rho\left(\left|D \varphi^{-}\right|^{2}\right) D \varphi^{-}\right) \quad \text { in } \mathbb{R}^{n} .
$$

Since $\varphi^{-}(x)$ satisfies $(1.1)$ in $\mathbb{R}^{n-1} \times(-1,1)$, then, from (3.1), we have that $g$ satisfies

$$
\begin{aligned}
& g \in C^{\alpha}\left(\mathbb{R}^{n}\right), \quad\|g\|_{0, \alpha, \mathbb{R}^{n}}^{(n+1)} \leq C \sigma, \\
& \operatorname{supp}(g) \subset\left(\mathbb{R}^{n-1} \times(1,2)\right) \cup\left(\mathbb{R}^{n-1} \times(-2,-1)\right) .
\end{aligned}
$$

Define

$$
F\left(x^{\prime}, x_{n}\right)=\int_{0}^{x_{n}} g\left(x^{\prime}, s\right) d s \text { in } \mathbb{R}^{n} .
$$

Then, from (3.4) and (3.5), we have

$$
\begin{aligned}
& F, F_{x_{n}} \in C^{\alpha}\left(\mathbb{R}^{n}\right), \\
& \|F\|_{0, \alpha, \mathbb{R}^{n-1} \times(-1,1)}^{(n+1)}+\left\|F_{x_{n}}\right\|_{0, \alpha, \mathbb{R}^{n}}^{(n+1)} \leq C \sigma, \\
& \sup _{\left(x^{\prime}, x_{n}\right) \in \mathbb{R}^{n}}\left(\left(1+\left|x^{\prime}\right|\right)^{n+1}\left|F\left(x^{\prime}, x_{n}\right)\right|\right) \leq C \sigma, \\
& F \equiv 0 \text { in } \mathbb{R}^{n-1} \times(-1,1) .
\end{aligned}
$$

From now on, we use the extended function $\varphi^{-}=\varphi^{-}(x)$, and $C$ may denote a different constant at each occurrence, depending only on the data, i.e., on $n$, $\gamma, \alpha,\left|V_{0}^{\prime}\right|$, and $q_{0}^{+}$, unless otherwise is specified. 


\section{2. - Free boundary problems}

Similarly to [6], [7], we first reformulate Problem A into a free boundary problem. The main reason is to replace the pointwise gradient condition in the definition $\Omega^{+}=\left\{x \in \Omega:|D \varphi(x)| \leq c_{*}\right\}$ by a condition involving $\varphi$ but not its derivatives. We first note that, for the unperturbed solution (2.14), we have $\varphi_{0} \leq \varphi_{0}^{-}$in $\Omega$, and $\left\{x \in \Omega:\left|D \varphi_{0}(x)\right| \leq c_{*}\right\}=\left\{x \in \Omega: \varphi_{0}(x)<\varphi_{0}^{-}(x)\right\}$. Since $\partial_{v}\left(\varphi_{0}^{-}-\varphi_{0}^{-}\right)>0$ on $S_{0}=\partial\left\{x \in \Omega: \varphi_{0}(x)<\varphi_{0}^{-}(x)\right\}$, we can expect that the same properties will hold for $\varphi$ which is a small perturbation of $\varphi_{0}$. This motivates

Problem B. Find $\varphi \in C\left(\mathbb{R}^{n}\right)$ and $f \in C^{2, \alpha}\left(\mathbb{R}^{n-1}\right)$ such that

(i) In $\mathbb{R}^{n}$,

$$
\varphi \leq \varphi^{-}
$$

(ii) $\varphi \in C^{2, \alpha}\left(\overline{\Omega^{+}}\right)$with $\Omega^{+}=\left\{\varphi<\varphi^{-}\right\}$, the noncoincidence set;

(iii) $\varphi$ is a solution of (1.1) in $\Omega^{+}$;

(iv) The free boundary $S=\partial \Omega^{+}$is given by the equation $x_{n}=f\left(x^{\prime}\right)$ for $x^{\prime} \in \mathbb{R}^{n-1}$ so that $\Omega^{+}=\left\{x_{n}>f\left(x^{\prime}\right)\right\}$;

(v) The free boundary condition (2.3) holds on $S$.

Note that Problem B is not equivalent to Problem A in general, but a solution of Problem B satisfying (2.19), (2.20), and (2.21) is a solution of Problem A, provided that $\sigma$ is sufficiently small.

\section{3. - Partial hodograph transform}

We attempt to find a solution $\varphi$ of Problem B, which satisfies (2.19)-(2.21). Let $\varphi(x)$ be such a solution. Define a function $u$ in $\Omega^{+}$by

$$
u(x)=\varphi^{-}(x)-\varphi(x) .
$$

Then (2.19) and (3.1) imply

$$
\left\|u-\left(q_{0}^{-}-q_{0}^{+}\right) x_{n}\right\|_{2, \alpha, \Omega^{+}}^{(n-2)} \leq C \sigma .
$$

In particular, if $\sigma$ is sufficiently small,

$$
0<\frac{q_{0}^{-}-q_{0}^{+}}{2} \leq u_{x_{n}}(x) \leq 2\left(q_{0}^{-}-q_{0}^{+}\right) \quad \text { for any } \quad x \in \Omega^{+} .
$$

Now we show that $u(x)$ is a solution of a boundary value problem for a uniformly elliptic equation. From (2.19) with sufficiently small $\sigma, \varphi(x)$ satisfies (1.1) in $\Omega^{+}$and (2.4) on $S$. Then, using (3.3), we see that $u(x)$ is a solution of the following problem:

$$
\begin{array}{ll}
\operatorname{div}(A(x, D u))=-g & \text { in } \Omega^{+}, \\
A(x, D u) \cdot v=0 & \text { on } S,
\end{array}
$$


where

$$
A(x, P)=\rho\left(\left|D \varphi^{-}(x)-P\right|^{2}\right)\left(D \varphi^{-}(x)-P\right)-\rho\left(\left|D \varphi^{-}(x)\right|^{2}\right) D \varphi^{-}(x)
$$

for $x \in \Omega^{+}$and $P \in \mathbb{R}^{n}$. Note that, from (2.20) and (2.21), for sufficiently small $\sigma$, the free boundary $S$ lies within the domain $\Omega_{1}=\mathbb{R}^{n-1} \times(-1,1)$. Then, by (3.6), the function $F$ defined by (3.5) vanishes on $S$, and thus we can rewrite (3.10) as the following conormal boundary value problem:

$$
\begin{array}{ll}
\operatorname{div}\left(A(x, D u)+F(x) e_{n}\right)=0 & \text { in } \quad \Omega^{+}, \\
\left(A(x, D u)+F(x) e_{n}\right) \cdot v=0 & \text { on } \quad S,
\end{array}
$$

where $e_{n}=(0, \ldots, 0,1)$. Equation (3.12) is uniformly elliptic for $u$ if $\sigma$ is sufficiently small, which follows from (2.16) and (3.8) since

$$
0<c_{0} \leq \Phi^{\prime}(q)=\rho\left(q^{2}\right)+2 q^{2} \rho^{\prime}\left(q^{2}\right) \leq C \quad \text { for } q \text { near } q_{0}^{+},
$$

for some constants $c_{0}$ and $C>0$. Note that the weak form of problem (3.12)(3.13) is

$$
\int_{\Omega^{+}}\left(A(x, D u)+F(x) e_{n}\right) \cdot D \eta d x=0 \quad \text { for any } \eta \in C_{0}^{1}\left(\mathbb{R}^{n}\right) .
$$

Since $\varphi=\varphi^{-}$on $S$, it follows that

$$
u=0 \quad \text { on } S .
$$

Now we make a change of variables. Define a mapping $\Phi: \overline{\Omega^{+}} \rightarrow \mathbb{R}^{n}$ by

$$
\left(x^{\prime}, x_{n}\right) \rightarrow\left(y^{\prime}, y_{n}\right)=\left(x^{\prime}, u\left(x^{\prime}, x_{n}\right)\right) .
$$

The nondegeneracy property (3.9) implies that the map $\Phi$ is one-to-one on $\overline{\Omega^{+}}$ and, from (2.20), (3.9), and (3.15),

$$
\Phi\left(\Omega^{+}\right)=\mathbb{R}_{+}^{n}, \quad \Phi(S)=\partial \mathbb{R}_{+}^{n},
$$

i.e., the free boundary $S$ is mapped to the fixed boundary $\partial \mathbb{R}_{+}^{n}$. Also, by (3.9), there exists a function $v \in C^{2, \alpha}\left(\overline{\mathbb{R}_{+}^{n}}\right)$ such that, for $\left(x^{\prime}, x_{n}\right) \in \overline{\Omega^{+}}$and $y_{n} \geq 0$,

$$
u\left(x^{\prime}, x_{n}\right)=y_{n} \quad \text { if and only if } \quad v\left(x^{\prime}, y_{n}\right)=x_{n} .
$$

Thus

$$
\Phi^{-1}\left(y^{\prime}, y_{n}\right)=\left(y^{\prime}, v\left(y^{\prime}, y_{n}\right)\right) .
$$

Differentiating the identity $u\left(x^{\prime}, v\left(x^{\prime}, y_{n}\right)\right)=y_{n}$, which holds for any $\left(x^{\prime}, y_{n}\right) \in$ $\overline{\mathbb{R}_{+}^{n}}$, we find

$$
v_{y_{n}}>0 \quad \text { in } \mathbb{R}_{+}^{n},
$$


and

$$
D_{x^{\prime}} u=-\frac{1}{v_{y_{n}}} D_{y^{\prime}} v, \quad u_{x_{n}}=\frac{1}{v_{y_{n}}},
$$

where the left-hand and right-hand sides are taken at the points $\left(x^{\prime}, x_{n}\right)$ and $\Phi\left(x^{\prime}, x_{n}\right)$, respectively. In particular, (3.9) implies

$$
0<\frac{1}{2\left(q_{0}^{-}-q_{0}^{+}\right)} \leq v_{y_{n}} \leq \frac{2}{q_{0}^{-}-q_{0}^{+}} \quad \text { for any } \quad y \in \mathbb{R}_{+}^{n} .
$$

From this and (2.19), we get

$$
\left\|v-v_{0}\right\|_{2, \alpha, \mathbb{R}_{+}^{n}}^{(n-2)} \leq C \sigma
$$

where

$$
v_{0}(y)=\frac{y_{n}}{q_{0}^{-}-q_{0}^{+}} .
$$

Since $u(x)$ is a solution of the conormal boundary value problem (3.12)-(3.13) in $\Omega^{+}$, then $v(y)$ is a solution of the corresponding problem in $\mathbb{R}_{+}^{n}$. In order to show that this problem has also a conormal structure, we make the change of variables $x \rightarrow y=\Phi(x)$ in the weak form (3.14) of problem (3.12)-(3.13). In order to do that, we especially need to change the variables in the test function $\eta$. For that, we note that the function $\psi(y):=\eta \circ \Phi^{-1}(y)=\eta\left(y^{\prime}, v\left(y^{\prime}, y_{n}\right)\right)$ satisfies $\psi \in C^{1}\left(\overline{\mathbb{R}_{+}^{n}}\right)$ and, if $\eta \equiv 0$ on $\mathbb{R}^{n} \backslash B_{R}$, then $\psi \equiv 0$ on $\mathbb{R}_{+}^{n} \backslash B_{R_{1}}$ for some $R_{1}$, i.e., $\psi=\left.\tilde{\psi}\right|_{\mathbb{R}_{+}^{n}}$ for some $\tilde{\psi} \in C_{0}^{1}\left(\mathbb{R}^{n}\right)$. Similarly, for any $\psi \in C_{0}^{1}\left(\mathbb{R}^{n}\right)$, there exists $\eta \in C_{0}^{1}\left(\mathbb{R}^{n}\right)$ such that $\left.\psi\right|_{\mathbb{R}_{+}^{n}}=\eta \circ \Phi^{-1}$ and $\eta(x)=\psi \circ \Phi(x)$ for $x \in \overline{\Omega^{+}}$. We differentiate the identity $\eta(x)=\psi\left(x^{\prime}, u\left(x^{\prime}, x_{n}\right)\right)$ and use (3.17) to obtain

$$
D_{x^{\prime}} \eta=D_{y^{\prime}} \psi-\frac{\psi_{y_{n}}}{v_{y_{n}}} D_{y^{\prime}} v, \quad \eta_{y_{n}}=\frac{\psi_{y_{n}}}{v_{y_{n}}}
$$

Now, in (3.14), we make the change of variables $x \rightarrow y=\Phi(x)$, use (3.17) and (3.21), note that the Jacobian of $\Phi^{-1}$ is $J\left(\Phi^{-1}(y)\right)=v_{y_{n}}(y)$, and write $A\left(x^{\prime}, x_{n}, p^{\prime}, p_{n}\right)$ for $A(x, P)$ and $F\left(x^{\prime}, x_{n}\right)$ for $F(x)$ to obtain

$$
\int_{\mathbb{R}_{+}^{n}}\left(A\left(y^{\prime}, v,-\frac{1}{v_{y_{n}}} D_{y^{\prime}} v, \frac{1}{v_{y_{n}}}\right)+F\left(y^{\prime}, v\right) e_{n}\right) \cdot\left(\begin{array}{l}
v_{y_{n}} D_{y^{\prime}} \psi-\psi_{y_{n}} D_{y^{\prime}} v \\
\psi_{y_{n}}
\end{array}\right) d y=0
$$

for any $\psi \in C_{0}^{1}\left(\mathbb{R}^{n}\right)$. This can be written as

$$
\int_{\mathbb{R}_{+}^{n}}\left(B\left(y^{\prime}, v, D v\right)+F\left(y^{\prime}, v\right) e_{n}\right) \cdot D \psi d y=0 \quad \text { for any } \psi \in C_{0}^{1}\left(\mathbb{R}^{n}\right),
$$


where, for $y^{\prime} \in \mathbb{R}^{n-1}, z \in \mathbb{R}, P=\left(p^{\prime}, p_{n}\right) \in \mathbb{R}^{n-1} \times \mathbb{R}_{+}$,

$$
\begin{aligned}
& B^{i}\left(y^{\prime}, z, P\right)=A^{i}\left(y^{\prime}, z,-\frac{p^{\prime}}{p_{n}}, \frac{1}{p_{n}}\right) p_{n} \quad \text { for } \quad i=1, \ldots, n-1, \\
& B^{n}\left(y^{\prime}, z, P\right)=A^{n}\left(y^{\prime}, z,-\frac{p^{\prime}}{p_{n}}, \frac{1}{p_{n}}\right)-\sum_{i=1}^{n-1} A^{i}\left(y^{\prime}, z,-\frac{p^{\prime}}{p_{n}}, \frac{1}{p_{n}}\right) p_{i} .
\end{aligned}
$$

Thus, $v(y)$ satisfies the conormal boundary value problem:

$$
\begin{array}{ll}
\operatorname{div}\left(B\left(y^{\prime}, v, D v\right)+F\left(y^{\prime}, v\right) e_{n}\right)=0 & \text { in } \mathbb{R}_{+}^{n}, \\
B^{n}\left(y^{\prime}, v, D v\right)+F\left(y^{\prime}, v\right)=0 & \text { on } \partial \mathbb{R}_{+}^{n} .
\end{array}
$$

Conversely, let $v(y)$ is a solution of (3.24)-(3.25) satisfying (3.19) with $C \sigma$ sufficiently small, depending only on the data so that (3.18) holds. Then a function $u(x)$ can be defined on

$$
\Omega^{+}:=\left\{\left(x^{\prime}, v\left(x^{\prime}, y_{n}\right)\right): x^{\prime} \in \mathbb{R}^{n-1}, y_{n}>0\right\}
$$

such that (3.16) holds. Clearly, $u(x)$ satisfies (3.8) and (3.9). Making the change of variables $x=\Phi^{-1}(y)$ in (3.22), we see that $u(x)$ satisfies (3.14) and thus (3.12)-(3.13). Then

$$
\varphi(x):= \begin{cases}\varphi_{0}(x)-u(x) & \text { for } x \in \Omega^{+}, \\ \varphi_{0}(x) & \text { otherwise }\end{cases}
$$

is continuous in $\mathbb{R}^{n}$ and satisfies (2.19). Thus, $\varphi(x)$ is a solution of Problem $\mathrm{A}$ if $\sigma$ is so small that (2.19) implies that $\varphi$ is subsonic in $\Omega^{+}$. Moreover, from (3.18) and (3.26), $\Omega^{+}$satisfies (2.20) with $f\left(x^{\prime}\right)=v\left(x^{\prime}, 0\right)$. Thus, from (3.19), it follows that (2.21) holds.

Furthermore, assume $\sigma$ is small, depending only on the data, and $\varphi_{1}$ and $\varphi_{2}$ are two solutions of Problem A satisfying (2.19). Then both functions $u_{k}=\varphi^{-}-\varphi_{k}, k=1,2$, satisfy $\partial_{x_{n}} u_{k} \geq\left(q_{0}^{-}-q_{0}^{+}\right) / 2>0$, and thus functions $v_{k} \in C^{2, \alpha}\left(\overline{\mathbb{R}_{+}^{n}}\right), k=1,2$, are defined by (3.16) and satisfy (3.19) with $C$ depending only on the data. Note that $v_{1}$ is not identically equal to $v_{2}$ if $\varphi_{1}$ is not identically equal to $\varphi_{2}$.

Therefore, we have

Proposition 3.1. Assume that $\sigma$ is small, depending only on the data. Let $\varphi^{-}(x)$ be a supersonic solution of (1.1) satisfying the conditions stated in Problem A. Assume that problem (3.24)-(3.25), defined by (3.11) and (3.23), has a unique solution $v \in C^{2, \alpha}\left(\overline{\mathbb{R}_{+}^{n}}\right)$ satisfying (3.19). Then there exists a unique solution $\varphi$ of Problem A satisfying (2.19). Moreover, (2.20) and (2.21) hold for $\varphi$, and the function $u:=\varphi^{-}-\varphi$ is related with $v$ by (3.16). 


\section{4. - Solutions of the conormal boundary value problems}

By Proposition 3.1, in order to solve Problem A, it suffices to establish the existence and uniqueness for the conormal boundary value problem (3.24)-(3.25) satisfying (3.19). First, we show that (3.24) is elliptic in a neighborhood of the function $v_{0}(y)$ defined by (3.20), that is, there exist $\Lambda>\lambda>0$ such that

$$
\lambda|\xi|^{2} \leq \sum_{i, j=1}^{n} B_{p_{j}}^{i}\left(y^{\prime}, z, P\right) \xi_{i} \xi_{j} \leq \Lambda|\xi|^{2}
$$

for any $\xi \in \mathbb{R}^{n}$. From (3.23), we compute

$$
\sum_{i, j=1}^{n} B_{p_{j}}^{i}\left(y^{\prime}, z, P\right) \xi_{i} \xi_{j}=\sum_{i, j=1}^{n} A_{p_{j}}^{i}\left(y^{\prime}, z,-\frac{p^{\prime}}{p_{n}}, \frac{1}{p_{n}}\right) \zeta_{i} \zeta_{j},
$$

where

$$
\zeta_{i}=\xi_{i}-\frac{p_{i}}{p_{n}} \xi_{n}, \quad i=1, \ldots, n-1 ; \quad \zeta_{n}=\frac{1}{p_{n}} \xi_{n} .
$$

Since (3.12) is a uniformly elliptic equation for $u$ satisfying (3.8) with small $\sigma$, it follows that, if $P$ is sufficiently close to $D v_{0}=\frac{1}{q_{0}^{-}-q_{0}^{+}} e_{n}$, then (4.1) holds with the constants depending only on the data.

We will modify $B\left(y^{\prime}, z, P\right)$ away from a neighborhood of $\left(y, v_{0}(y), D v_{0}\right)$ to obtain a uniformly elliptic equation globally.

Note that $v_{0}(y)$ is a solution of the problem of form (3.24)-(3.25) with $B_{0}(P)$ which corresponds to the supersonic solution $\varphi_{0}^{-}(y)$, i.e., $B_{0}(P)$ is defined by (3.23) with $A_{0}(P)$, defined by (3.11) with $\varphi_{0}^{-}$instead of $\varphi^{-}$. Then $F_{0} \equiv 0$, and $A_{0}$ and $B_{0}$ depend only on $P$ since $\varphi_{0}^{-}$is a linear function.

Since we are interested in estimate (3.19), we introduce the function

$$
w(y)=v(y)-v_{0}(y)
$$

and rewrite (3.24)-(3.25) in terms of $w$. Using the fact that $v_{0}(y)$ is a solution of the conormal boundary value problem defined by $B_{0}(P)$, we find that $w(y)$ satisfies

$$
\begin{aligned}
& \operatorname{div}(\hat{\mathcal{N}}(y, w, D w))=0 \quad \text { in } \quad \mathbb{R}_{+}^{n}, \\
& \hat{\mathcal{N}}^{n}(y, w, D w)=0 \quad \text { on } \partial \mathbb{R}_{+}^{n},
\end{aligned}
$$

where $\hat{\mathcal{N}}(y, z, P)=B\left(y^{\prime}, v_{0}(y)+z, D v_{0}+P\right)-B_{0}\left(D v_{0}\right)+F\left(y^{\prime}, v_{0}(y)+z\right) e_{n}$. From the ellipticity of $B\left(y^{\prime}, z, P\right)$, it follows that (4.1) holds for $\hat{\mathcal{N}}(y, z, P)$ with the same ellipticity constants, if $|P|$ is sufficiently small.

Now we define a function $\mathcal{N}(y, z, P)$ as a modification of $\hat{\mathcal{N}}(y, z, P)$. Let nonnegative $\zeta, \eta \in C^{\infty}\left(\overline{\mathbb{R}_{+}}\right)$be such that

$$
\zeta(t)=\left\{\begin{array}{ll}
1 & \text { for } t<1, \\
0 & \text { for } t>2,
\end{array} \quad \eta(t)=\zeta\left(\frac{t}{\varepsilon}\right)\right.
$$


where the small constant $\varepsilon>0$ will be chosen below. Introduce the following notations

$X(y, z, P):=\left(y^{\prime}, v_{0}(y)+z, D v_{0}+P\right), \quad L_{0}(P):=B_{0}\left(D v_{0}\right)+D_{P} B_{0}\left(D v_{0}\right) \cdot P$.

Now we define the modification of $\hat{\mathcal{N}}(y, z, P)$ :

$$
\begin{aligned}
\mathcal{N}(y, z, P)= & D_{P} B_{0}\left(D v_{0}\right) \cdot P+\eta(|P|)\left(B(X(y, z, P))-L_{0}(P)\right) \\
& +F\left(y^{\prime}, v_{0}(y)+z\right) e_{n}
\end{aligned}
$$

Note that

$$
\mathcal{N}(y, z, P)=\hat{\mathcal{N}}(y, z, P) \quad \text { if } \quad|P|<\varepsilon
$$

We will also use the function

$$
\mathcal{M}(y, z, P)=D_{P} B_{0}\left(D v_{0}\right) \cdot P+\eta(|P|)\left(B(X(y, z, P))-L_{0}(P)\right) .
$$

Obviously, $\mathcal{N}(y, z, P)=\mathcal{M}(y, z, P)+F\left(y^{\prime}, v_{0}(y)+z\right) e_{n}$. We note the following properties of $\mathcal{N}(y, z, P)$ and $\mathcal{M}(y, z, P)$.

Proposition 4.1. There exist $\varepsilon_{0}, \sigma_{0}$, and $\Lambda \geq \lambda>0$ depending only on the data such that, if $\varepsilon=\varepsilon_{0}$ in (4.4) and $\sigma \leq \sigma_{0}$, then

(i) $\mathcal{N}$ is uniformly elliptic:

(4.6) $\lambda|\xi|^{2} \leq \sum_{i, j=1}^{n} \mathcal{N}_{p_{j}}^{i}(y, z, P) \xi_{i} \xi_{j} \leq \Lambda|\xi|^{2} \quad$ for every $y \in \overline{\mathbb{R}_{+}^{n}}, \quad z \in \mathbb{R}, P, \xi \in \mathbb{R}^{n}$

(ii) The following estimates hold:

$$
\begin{aligned}
& \left|\mathcal{N}\left(\left(y^{\prime}, y_{n}\right), z, P\right)\right|+\left|\mathcal{M}\left(\left(y^{\prime}, y_{n}\right), z, P\right)\right| \leq C\left(\frac{\sigma}{1+\left|y^{\prime}\right|^{n}}+|P|\right) \\
& \sum_{i, j=1}^{n}\left|\mathcal{N}_{p_{j}}^{i}(y, z, P)-B_{0 p_{j}}^{i}\left(D v_{0}\right)\right| \leq C\left(\frac{\sigma \varepsilon_{0}^{-1}}{1+\left|y+z e_{n}\right|^{n}}+|P|\right) \chi_{\left[0,2 \varepsilon_{0}\right]}(|P|), \\
& \left|\mathcal{N}_{z}(y, z, P)\right|+\sum_{i=1}^{n-1} \sum_{j=1}^{n}\left|\mathcal{N}_{y_{j}}^{i}(y, z, P)\right|+\left|\mathcal{N}_{y_{n}}^{n}(y, z, P)\right| \\
& \quad \leq \frac{C \sigma}{1+\left|y+z e_{n}\right|^{n+1}} \chi_{\left[0,2 \varepsilon_{0}\right]}(|P|)
\end{aligned}
$$

$$
\mathcal{N}_{z} \in C\left(\overline{\mathbb{R}_{+}^{n}} \times \mathbb{R} \times \mathbb{R}^{n}\right),
$$




$$
\begin{gathered}
\left|\mathcal{M}_{z}(y, z, P)\right|+\sum_{i, j=1}^{n}\left|\mathcal{M}_{y_{j}}^{i}(y, z, P)\right| \leq \frac{C \sigma}{1+\left|y+z e_{n}\right|^{n+1}} \chi_{\left[0,2 \varepsilon_{0}\right]}(|P|), \\
\left|\mathcal{M}_{z}(y, z, P)-\mathcal{M}_{z}(\tilde{y}, \tilde{z}, P)\right|+\sum_{i, j=1}^{n}\left|\mathcal{M}_{y_{j}}^{i}(y, z, P)-\mathcal{M}_{y_{j}}^{i}(\tilde{y}, \tilde{z}, P)\right| \\
\leq \frac{C \sigma}{1+\left(\min \left(\left|y+z e_{n}\right|,\left|\tilde{y}+\tilde{z} e_{n}\right|\right)\right)^{n+1+\alpha}}\left(|y-\tilde{y}|^{2}+|z-\tilde{z}|^{2}\right)^{\alpha / 2},
\end{gathered}
$$

for every $y \in \overline{\mathbb{R}_{+}^{n}}, z \in \mathbb{R}$, and $P \in \mathbb{R}^{n}$, where $\chi_{\left[0,2 \varepsilon_{0}\right]}(\cdot)$ is the characteristic function of the interval $\left[0,2 \varepsilon_{0}\right], D v_{0}=\frac{1}{q_{0}^{-}-q_{0}^{+}} e_{n}$, and the constant $C$ depends only on the data and is independent of $\varepsilon_{0}$.

Moreover, the following estimates hold:

$$
\begin{aligned}
& \left|D_{P}^{2} \mathcal{N}(y, z, P)\right|+\left|D_{P}^{3} \mathcal{N}(y, z, P)\right|=\left|D_{P}^{2} \mathcal{M}(y, z, P)\right|+\left|D_{P}^{3} \mathcal{M}(y, z, P)\right| \\
& \quad \leq C\left(\frac{\sigma}{1+\left|y+z e_{n}\right|^{n}}+|P|\right) \chi_{\left[0,2 \varepsilon_{0}\right]}(|P|),
\end{aligned}
$$

$$
\begin{gathered}
\left|D_{y P}^{2} \mathcal{M}(y, z, P)\right|+\left|D_{z P}^{2} \mathcal{M}(y, z, P)\right| \leq \frac{C \sigma}{1+\left|y+z e_{n}\right|^{n+1}} \chi_{\left[0,2 \varepsilon_{0}\right]}(|P|) \\
\left|D_{y P}^{2} \mathcal{M}(y, z, P)-D_{y P}^{2} \mathcal{M}(\tilde{y}, \tilde{z}, P)\right|+\left|D_{z P}^{2} \mathcal{M}(y, z, P)-D_{z P}^{2} \mathcal{M}(\tilde{y}, \tilde{z}, P)\right| \\
\leq \frac{C \sigma}{1+\left(\min \left(\left|y+z e_{n}\right|,\left|\tilde{y}+\tilde{z} e_{n}\right|\right)\right)^{n+1+\alpha}}\left(|y-\tilde{y}|^{2}+|z-\tilde{z}|^{2}\right)^{\alpha / 2}
\end{gathered}
$$

for every $y \in \overline{\mathbb{R}_{+}^{n}}, z \in \mathbb{R}$, and $P \in \mathbb{R}^{n}$, where the constant $C$ depends only on the data and $\varepsilon_{0}$.

Proof. We first prove (4.7). Denote

$$
M=\sup \left\{\left|D\left(\rho\left(|Q|^{2}\right) Q\right)\right|: Q \in \mathbb{R}^{n},|Q|<\left|D \varphi_{0}^{-}\right|+2 \varepsilon_{0}+\sigma_{0}\right\} .
$$

Clearly, $M$ depends only on the data. If $\varepsilon_{0} \leq \frac{\left|D v_{0}\right|}{8}=\frac{1}{8\left(q_{0}^{-}-q_{0}^{+}\right)}$, we get for $y=\left(y^{\prime}, y_{n}\right)$,

$$
\begin{aligned}
|\mathcal{N}(y, z, P)| \leq & \left|B(X(y, z, P))-B_{0}\left(D v_{0}\right)\right| \chi_{\left[0,2 \varepsilon_{0}\right]}(|P|)+(1-\eta(|P|))\left|D_{P} B_{0}\left(D v_{0}\right) \cdot P\right| \\
& +\left|F\left(y^{\prime}, v_{0}(y)+z\right)\right| \\
\leq & M\left|D \varphi^{-}\left(y^{\prime}, z\right)-D \varphi_{0}^{-}\left(y^{\prime}, z\right)\right| \chi_{\left[0,2 \varepsilon_{0}\right]}(|P|)+C|P|+\left|F\left(y^{\prime}, v_{0}(y)+z\right)\right| \\
\leq & C\left(\frac{\sigma}{1+\left|y+z e_{n}\right|^{n}} \chi_{\left[0,2 \varepsilon_{0}\right]}(|P|)+|P|+\frac{\sigma}{1+\left|y^{\prime}\right|^{n+1}}\right) \\
\leq & C\left(\frac{\sigma}{1+\left|y^{\prime}\right|^{n}}+|P|\right) .
\end{aligned}
$$


The estimate of $|\mathcal{M}(y, z, P)|$ involves only the terms that do not contain $F$ in the above inequalities, and thus we obtain the same estimate. Now (4.7) is proved.

From the definition,

$$
\begin{aligned}
\mathcal{N}_{p_{j}}^{i}(y, z, P)= & B_{0 p_{j}}^{i}\left(D v_{0}\right)+\eta^{\prime}(|P|) \frac{p_{j}}{|P|}\left(B^{i}(X(y, z, P))-\left(L_{0}\right)^{i}(P)\right) \\
& +\eta(|P|)\left(B_{p_{j}}^{i}(X(y, z, P))-\left(L_{0}\right)_{p_{j}}^{i}(P)\right) \\
= & B_{0 p_{j}}^{i}\left(D v_{0}\right)+A_{1}+A_{2} .
\end{aligned}
$$

If $\varepsilon_{0} \leq \frac{\left|D v_{0}\right|}{8}=\frac{1}{8\left(q_{0}^{-}-q_{0}^{+}\right)}$, we use (2.16), (3.11), (3.20), (3.23), and (4.2) to obtain

$$
\begin{aligned}
\left|A_{1}\right| & \leq \chi_{\left[0,2 \varepsilon_{0}\right]}(|P|) \frac{C}{\varepsilon_{0}}\left(\left|B^{i}(X(y, z, P))-B_{0}^{i}\left(D v_{0}+P\right)\right|+\left|B_{0}^{i}\left(D v_{0}+P\right)-\left(L_{0}\right)^{i}(P)\right|\right) \\
& \leq \chi_{\left[0,2 \varepsilon_{0}\right]}(|P|) \frac{C}{\varepsilon_{0}}\left(\left|D \varphi^{-}\left(y^{\prime}, v_{0}(y)+z\right)-D \varphi_{0}^{-}\left(y^{\prime}, v_{0}(y)+z\right)\right|+|P|^{2}\right) \\
& \leq \chi_{\left[0,2 \varepsilon_{0}\right]}(|P|) \frac{C}{\varepsilon_{0}}\left(\frac{\sigma}{1+\left|y+z e_{n}\right|^{n}}+\varepsilon_{0}|P|\right) \leq C \chi_{\left[0,2 \varepsilon_{0}\right]}(|P|)\left(\frac{\sigma \varepsilon_{0}^{-1}}{1+\left|y+z e_{n}\right|^{n}}+|P|\right)
\end{aligned}
$$

and

$$
\begin{aligned}
&\left|A_{2}\right| \leq C \chi_{\left[0,2 \varepsilon_{0}\right]}(|P|)\left(\left|B_{p_{j}}^{i}(X(y, z, P))-B_{0 p_{j}}^{i}\left(D v_{0}+P\right)\right|\right. \\
&\left.+\left|B_{0 p_{j}}^{i}\left(D v_{0}+P\right)-B_{0 p_{j}}^{i}\left(D v_{0}\right)\right|\right) \\
& \leq C \chi_{\left[0,2 \varepsilon_{0}\right]}(|P|)\left(\left|D \varphi^{-}\left(y^{\prime}, v_{0}(y)+z\right)-D \varphi_{0}^{-}\left(y^{\prime}, v_{0}(y)+z\right)\right|+|P|\right) \\
& \leq C \chi_{\left[0,2 \varepsilon_{0}\right]}(|P|)\left(\frac{\sigma}{1+\left|y+z e_{n}\right|^{n}}+|P|\right) .
\end{aligned}
$$

This proves (4.8).

Now we prove (4.9). Note that

(4.16) $\mathcal{N}_{z}^{n}(y, z, P)=\eta(|P|) B_{z}^{n}(X(y, z, P))+F_{x_{n}}\left(y^{\prime}, v_{0}(y)+z\right)=A_{3}+A_{4}$.

We estimate

$$
\begin{aligned}
\left|A_{3}\right| & \leq C \chi_{\left[0,2 \varepsilon_{0}\right]}(|P|)\left|B_{z}(X(y, z, P))\right| \\
& \leq C \chi_{\left[0,2 \varepsilon_{0}\right]}(|P|)\left|D^{2} \varphi^{-}\left(y^{\prime}, v_{0}(y)+z\right)\right| \\
& \leq C \chi_{\left[0,2 \varepsilon_{0}\right]}(|P|) \frac{\sigma}{1+\left|y+z e_{n}\right|^{n+1}},
\end{aligned}
$$

and, by (3.6),

$$
\left|A_{4}\right| \leq \frac{C \sigma}{1+\left|\left(y^{\prime}, v_{0}(y)+z\right)\right|^{n+1}} \leq \frac{C \sigma}{1+\left|y+z e_{n}\right|^{n+1}} .
$$


Thus, estimate (4.9) for $\mathcal{N}_{z}^{n}$ is proved. The term $\mathcal{N}_{y_{n}}^{n}$ is estimated similarly, since $v_{0}(y)=y_{n} /\left(q_{0}^{-}-q_{0}^{+}\right)$. For $i=1, \ldots, n-1$ and $j=1, \ldots, n$, we have $\mathcal{N}_{z}^{i}(y, z, P)=\eta(|P|) B_{z}^{i}(X(y, z, P)), \quad \mathcal{N}_{y_{j}}^{i}(y, z, P)=d_{j} \eta(|P|) B_{y_{j}}^{i}(X(y, z, P))$, where $d_{j}=1$ for $j=1, \ldots, n-1$ and $d_{n}=\left(q_{0}^{-}-q_{0}^{+}\right)^{-1}$. Thus, $\mathcal{N}_{z}^{i}$ and $\mathcal{N}_{y_{j}}^{i}$, for $i=1, \ldots, n-1$ and $j=1, \ldots, n$, are estimated similar to the term $A_{3}$ above. Thus, (4.9) is proved.

Note that (4.11) is also proved, since (4.11) follows from the estimates of the term $A_{3}$ above, and these estimates hold for $\mathcal{M}_{z}^{i}$ and $\mathcal{M}_{y_{j}}^{i}$ for any $i, j=1, \ldots, n$.

Also, (4.16) implies (4.10) since all the terms on the right-hand side of (4.16) are continuous.

Now we prove (4.12). Notice that

$$
\begin{aligned}
& \left|\mathcal{M}_{z}(y, z, P)-\mathcal{M}_{z}(\tilde{y}, \tilde{z}, P)\right| \\
& \leq \eta(|P|)\left|B_{z}(X(y, z, P))-B_{z}(X(\tilde{y}, \tilde{z}, P))\right| \\
& \leq C\left|D^{2} \varphi^{-}\left(y^{\prime}, v_{0}(y)+z\right)-D^{2} \varphi^{-}\left(\tilde{y}_{2}^{\prime}, v_{0}(\tilde{y})+\tilde{z}\right)\right| \chi_{\left[0,2 \varepsilon_{0}\right]}(|z|) \\
& \leq \frac{C \sigma}{1+\left(\min \left(\left|y+z e_{n}\right|,\left|\tilde{y}+\tilde{z} e_{n}\right|\right)\right)^{n+1+\alpha}}\left(|y-\tilde{y}|^{2}+|z-\tilde{z}|^{2}\right)^{\alpha / 2} .
\end{aligned}
$$

We estimate $\left|\mathcal{N}_{y_{j}}^{i}(y, z, P)-\mathcal{N}_{y_{j}}^{i}(\tilde{y}, \tilde{z}, P)\right|$ for $i, j=1, \ldots, n$, similarly.

Estimates (4.13)-(4.15) are proved similarly to estimates (4.8)-(4.12), since the functions $\mathcal{M}$ and $D_{P} \mathcal{M}$ are of the same structure.

It remains to prove assertion (i). Since $B_{0}(P)$ satisfies the ellipticity condition (4.1) at $P=D v_{0}$ with ellipticity constants $\lambda$ and $\Lambda$ depending only on the data, then, from (4.8), choosing sufficiently small $\varepsilon_{0}$ and $\sigma_{0}:=\varepsilon_{0}^{2}$ yields (4.6) with ellipticity constants $\lambda / 2$ and $2 \Lambda$ for $\sigma \leq \sigma_{0}$,

From now on, we assume that $\varepsilon=\varepsilon_{0}$ is chosen in the definition of $\mathcal{N}$ and that $\sigma \leq \sigma_{0}$ so that Proposition 4.1 holds. Thus, in order to construct a solution of problem (3.24)-(3.25), it suffices to construct a solution of the problem

$$
\begin{array}{ll}
\operatorname{div}(\mathcal{N}(y, w, D w))=0 & \text { in } \mathbb{R}_{+}^{n}, \\
\mathcal{N}^{n}(y, w, D w)=0 & \text { on } \partial \mathbb{R}_{+}^{n},
\end{array}
$$

which is sufficiently small in an appropriate norm if $\sigma$ is small. In order to construct such a solution, we will construct solutions in bounded domains

$$
B_{R}^{+}:=\mathbb{R}_{+}^{n} \cap B_{R} \quad \text { with } \quad B_{R}=\{|x|<R\}
$$

and pass to the limit as $R \rightarrow \infty$. The main goal is now to obtain the estimates independent of $R$. More precisely, we consider following problems:

$$
\begin{array}{ll}
\operatorname{div}(\mathcal{N}(x, w, D w))=0 & \text { in } B_{R}^{+}, \\
\mathcal{N}^{n}(x, w, D w)=0 & \text { on } S^{R}:=\left\{x_{n}=0\right\} \cap B_{R}, \\
w=0 & \text { on } \partial B_{R} \cap \overline{\mathbb{R}_{+}^{n}} .
\end{array}
$$


Proposition 4.2. Let $w \in C\left(\overline{B_{R}^{+}}\right) \cap C^{2}\left(B_{R}^{+}\right)$be a solution of (4.19). Then, if $\sigma$ is sufficiently small,

$$
\|w\|_{0,0, B_{R}^{+}}^{(n-2)} \leq C \sigma
$$

ProOF. We prove this lemma by constructing a comparison function that is derived from the fundamental solution of the linear elliptic operator:

$$
\overline{\mathcal{L}} V=\sum_{i, j=1}^{n} B_{0 p_{j}}^{i}\left(D v_{0}\right) V_{x_{i} x_{j}}
$$

Let $D=\left[d_{i j}\right]$ be the inverse matrix of $\left[B_{0 p_{j}}^{i}\left(D v_{0}\right)\right]$. Then $D$ is symmetric and strictly positive definite. For $x \in \mathbb{R}^{n}$, denote

$$
|x|_{D}=\left(\sum_{i, j=1}^{n} d_{i j} x_{i} x_{j}\right)^{\frac{1}{2}}
$$

Then

$$
\frac{1}{\sqrt{\Lambda}}|x| \leq|x|_{D} \leq \frac{1}{\sqrt{\lambda}}|x| .
$$

In particular, fixing $\tau=1 / 2$, there exists $\mu>0$ depending only on $\Lambda$ and $\lambda$ such that

$$
|x|_{D}^{\tau}>4 \mu \quad \text { for } \quad|x|>1
$$

Consider the function

$$
V(x)=L\left(\frac{1}{\left|x^{*}\right|_{D}^{n-2}}-\frac{\mu}{\left|x^{*}\right|_{D}^{n-2+\tau}}\right) \quad \text { for } \quad x^{*}=x+e_{n},
$$

where the constant $L \in(0,1]$ will be chosen below.

From the choice of $\mu$, we have $V(x)>0$ for $x \in \mathbb{R}_{+}^{n}$. Since, for $x \in \mathbb{R}_{+}^{n}$, $\left|x^{*}\right|_{D}>c(\lambda)$ for any $x \in \overline{B_{R}^{+}}$, then, by choosing $L \in(0,1)$ small depending only on $\lambda$, we have $0<V<1$ in $\mathbb{R}_{+}^{n}$. In particular,

$$
\frac{1}{1+\left|x+V(x) e_{n}\right|} \leq \frac{2}{1+|x|} \quad \text { for any } \quad x \in \overline{B_{R}^{+}} \text {. }
$$

Note that

$$
\overline{\mathcal{L}} V=-L \mu \frac{\tau(n-2+\tau)}{\left|x^{*}\right|_{D}^{n+\tau}}
$$


Now we use (4.8), (4.9), and (4.23) to calculate

(4.25) $\operatorname{div}(\mathcal{N}(x, V, D V))=\sum_{i, j=1}^{n} \mathcal{N}_{p_{j}}^{i}(x, V, D V) V_{x_{i} x_{j}}$

$$
\begin{aligned}
& +\sum_{i=1}^{n} \mathcal{N}_{z}^{i}(x, V, D V) V_{x_{i}}+\sum_{i=1}^{n} \mathcal{N}_{y_{i}}^{i}(x, V, D V) \\
= & \overline{\mathcal{L}} V+\sum_{i, j=1}^{n}\left(\mathcal{N}_{p_{j}}^{i}(x, V, D V)-B_{0 p_{j}}^{i}\left(D v_{0}\right)\right) V_{x_{i} x_{j}} \\
& +\sum_{i=1}^{n}\left(\mathcal{N}_{z}^{i}(x, V, D V) V_{x_{i}}+\mathcal{N}_{y_{i}}^{i}(x, V, D V)\right) \\
\leq & -L \mu \frac{\tau(n-2+\tau)}{\left|x^{*}\right|_{D}^{n+\tau}}+\frac{L^{2} C}{\left|x^{*}\right|_{D}^{2 n-1}}+\frac{L C \sigma}{\left|x^{*}\right|_{D}^{n}\left(1+|x|^{n}\right)} \\
& +\frac{C \sigma}{\left|x^{*}\right|_{D}^{n-1}\left(1+|x|^{n+1}\right)}+\frac{C \sigma}{1+|x|^{n+1}} \\
\leq & -\frac{L \mu \tau(n-2+\tau)}{\left|x^{*}\right|_{D}^{n+\tau}}+\frac{L^{2} C}{\left|x^{*}\right|_{D}^{n+\tau}}+\frac{C L \sigma}{\left|x^{*}\right|_{D}^{n+\tau}}+\frac{C \sigma}{\left|x^{*}\right|_{D}^{n+\tau}},
\end{aligned}
$$

where $C$ depends only on the data, and we have used $n \geq 3, \tau=1 / 2$, and $\left|x^{*}\right|_{D} \leq C(\lambda)\left|x^{*}\right| \leq C(\lambda)(1+|x|)$ since $x_{n} \geq 0$. Choosing $L \in(0,1)$ small depending only on $n$ and the constant $C$ in the last estimate leads to

$$
\operatorname{div}(\mathcal{N}(x, V, D V)) \leq-\frac{L \mu \tau(n-2+\tau)}{2\left|x^{*}\right|_{D}^{n+\tau}}+\frac{C \sigma}{\left|x^{*}\right|_{D}^{n+\tau}}
$$

Next, we estimate the boundary operator on $\left\{x_{n}=0\right\}$ :

$$
\begin{aligned}
\mathcal{N}^{n}(x, V, D V)= & \mathcal{N}^{n}(x, V, 0)+\int_{0}^{1} \mathcal{N}_{p_{j}}^{i}(x, V, s D V) V_{x_{j}} v_{i} d s \\
= & \sum_{j=1}^{n}\left\{B_{0 p_{j}}^{n}\left(D v_{0}\right) V_{x_{j}}+\int_{0}^{1}\left(\mathcal{N}_{p_{j}}^{i}(x, V, s D V)-B_{0 p_{j}}^{n}\left(D v_{0}\right)\right) V_{x_{j}} d s\right\} \\
& +\mathcal{N}^{n}(x, V, 0) .
\end{aligned}
$$

Recalling that $D=\left[d_{i j}\right]$ is the inverse matrix of $\left[B_{0 p_{j}}^{i}\left(D v_{0}\right)\right]$, we get that, on $\left\{x_{n}=0\right\}$,

$$
\begin{aligned}
\sum_{j=1}^{n} B_{0 p_{j}}^{n}\left(D v_{0}\right) V_{x_{j}}(x) & =L \sum_{j, k=1}^{n} B_{0 p_{j}}^{n}\left(D v_{0}\right) d_{j k}\left(-(n-2) \frac{x_{k}^{*}}{\left|x^{*}\right|_{D}^{n}}+\mu(n-2+\tau) \frac{x_{k}^{*}}{\left|x^{*}\right|_{D}^{n+\tau}}\right) \\
& =L\left(-(n-2) \frac{1}{\left|x^{*}\right|_{D}^{n}}+\mu(n-2+\tau) \frac{1}{\left|x^{*}\right|_{D}^{n+\tau}}\right) \\
& \leq-\frac{L(n-2)}{2} \frac{1}{\left|x^{*}\right|_{D}^{n}},
\end{aligned}
$$


by (4.21) since $n \geq 3$ and $\tau=1 / 2$. Using this and estimates (4.7) (with $x=\left(x^{\prime}, 0\right)$, i.e., $|x|=\left|x^{\prime}\right|$ at the boundary), (4.8), and (4.23), we get from (4.27) that

$$
\begin{aligned}
\left.\mathcal{N}^{n}(x, V, D V)\right|_{x_{n}=0} & \leq-L \frac{(n-2)}{2} \frac{1}{\left|x^{*}\right|_{D}^{n}}+\left(\frac{C \sigma}{1+|x|^{n}}+|D V|\right)|D V|+\frac{C \sigma}{1+|x|^{n}} \\
& \leq L\left(-\frac{(n-2)}{2} \frac{1}{\left|x^{*}\right|_{D}^{n}}+\frac{C L}{\left|x^{*}\right|_{D}^{2 n-2}}\right)+\frac{C L \sigma}{\left|x^{*}\right|_{D}^{2 n-1}}+\frac{C \sigma}{\left|x^{*}\right|_{D}^{n}} \\
& \leq L\left(-\frac{(n-2)}{2} \frac{1}{\left|x^{*}\right|_{D}^{n}}+\frac{C L}{\left|x^{*}\right|_{D}^{n}}\right)+\frac{C L \sigma}{\left|x^{*}\right|_{D}^{n}}+\frac{C \sigma}{\left|x^{*}\right|_{D}^{n}} .
\end{aligned}
$$

Since $n \geq 3$, then choosing again $L \in(0,1)$ sufficiently small, depending only on $n$ and the constant $C$ in the last expression (i.e., on the data), we get

$$
\left.\mathcal{N}^{n}(x, V, D V)\right|_{x_{n}=0} \leq-L \frac{(n-2)}{4} \frac{1}{\left|x^{*}\right|_{D}^{n}}+\frac{C \sigma}{\left|x^{*}\right|_{D}^{n}} .
$$

Now $V(x)$ is a supersolution of the conormal boundary value problem if we choose $L \in(0,1)$ and $\sigma$ such that the right-hand sides in (4.26) and (4.28) are negative. Since $n \geq 3$ and $\tau=1 / 2$, the right-hand sides in (4.26) and (4.28) are negative if we choose

$$
L=C \sigma,
$$

where $C$ is sufficiently large depending only on $n, \mu$, and the constant $C$ on the right-hand sides in (4.26) and (4.28). Choosing $\sigma_{0}$ sufficiently small, we get that $L$ is sufficiently small to satisfy all the smallness requirements stated in the above argument, if $\sigma \leq \sigma_{0}$.

Now, by the comparison principle in Theorem B.1 (i) in Appendix (for which the ellipticity of $\mathcal{N},(4.9),(4.10)$, and (4.13) can be applied), we get $w \leq V$ in $\mathbb{R}_{+}^{n}$. Similar argument shows that $w \geq-V$ in $B_{R}^{+}$. Then

$$
|w| \leq V .
$$

Since

$$
|V| \leq \frac{C L}{1+|x|^{n-2}} \leq \frac{C \sigma}{1+|x|^{n-2}},
$$

where the last inequality follows from our choice of $L=C \sigma$, then the lemma is proved.

PRoposition 4.3. If $\sigma>0$ is sufficiently small, then, for any $R>1$, there exists a unique solution $w \in C\left(\overline{B_{R}^{+}}\right) \cap C^{2, \alpha}\left(\overline{B_{R}^{+}} \backslash\left(\partial B_{R} \cap\left\{x_{n}=0\right\}\right)\right)$ of (4.19) such that

$$
\|w\|_{2, \alpha, B_{R / 2}^{+}}^{(n-2)} \leq C \sigma,
$$

where $C$ depends only on the data and is independent of $R$. 
Proof. The existence of a solution $w \in C\left(\Omega_{R}\right) \cap C^{2, \alpha}\left(\overline{B_{R}^{+}} \backslash\left(\partial B_{R} \cap\left\{x_{n}=0\right\}\right)\right)$ of (4.19) follows by combining the theory of mixed boundary value problems for linear elliptic equations of [24] with the estimates for Dirichlet and oblique boundary value problems for nonlinear elliptic equations of [16] and [25]. Note, in particular, that the barrier construction of [24, Lemma 2] works for the nonlinear problem (4.19): the proof is a direct computation, similar to Proposition 4.2.

Thus, it suffices to prove estimate (4.29). We will prove (4.29) by rescaling.

First note that, by Proposition 4.2 for sufficiently small $\sigma$, we can rewrite (4.19) in the form:

$$
\begin{array}{ll}
\operatorname{div}(\mathcal{M}(x, w, D w))+g\left(x^{\prime}, v_{0}(x)+w\right)\left(\frac{1}{q_{0}^{-}-q_{0}^{+}}+w_{x_{n}}\right)=0 \text { in } B_{R}^{+}, \\
\mathcal{M}^{n}(x, w, D w)=0 \quad \text { on } S^{R}:=\left\{x_{n}=0\right\} \cap B_{R}, \\
w=0 \quad \text { on } \partial B_{R} \cap \overline{\mathbb{R}_{+}^{n}}
\end{array}
$$

where $\mathcal{M}$ and $g$ are defined by (4.5) and (3.3), respectively. Indeed, since $F\left(x^{\prime}, x_{n}\right) \equiv 0$ in $\mathbb{R}^{n-1} \times[-1,1]$ and by (3.20), it follows that, if $|w(x)| \leq \frac{1}{2}$ in $\mathbb{R}^{n-1} \times\left[-\frac{q_{0}^{-}-q_{0}^{+}}{2}, \frac{q_{0}^{-}-q_{0}^{+}}{2}\right]$, then

$$
F\left(x^{\prime}, v_{0}(x)+w(x)\right) \equiv 0 \quad \text { in } \mathbb{R}^{n-1} \times\left[-\frac{q_{0}^{-}-q_{0}^{+}}{2}, \frac{q_{0}^{-}-q_{0}^{+}}{2}\right]
$$

which can be achieved by (4.20) and choosing $\sigma$ small.

We can assume $R>4$. By Proposition 4.1 with the choice of $\varepsilon=\varepsilon_{0}$, the functions $\mathcal{M}(x, z, P)$ and $B(x, z, P):=g\left(x^{\prime}, v_{0}(x)+z\right)\left(\frac{1}{q_{0}^{-}-q_{0}^{+}}+p_{n}\right)$ satisfy the conditions of Theorem A.1 in $B_{1}^{+}$with the constants $\lambda, \Lambda$, and $M$ depending only on the data, and

$$
\|\mathcal{M}(\cdot, \cdot, 0)\|_{1, \alpha, B_{1}^{+} \times \mathbb{R}} \leq C \sigma, \quad\|B(\cdot, \cdot, 0)\|_{1, \alpha, B_{1}^{+} \times \mathbb{R}} \leq C \sigma .
$$

Then, using Proposition 4.2, we can apply Theorem A.1 in $B_{1}^{+}$to obtain

$$
\|w\|_{2, \alpha, B_{1 / 2}^{+}} \leq C\left(\|w\|_{0,0, B_{1}^{+}}+\sigma\right) \leq C \sigma .
$$

Thus, we get

$$
\|w\|_{2, \alpha, B_{1 / 2}^{+}}^{(n-2)} \leq C\|w\|_{2, \alpha, B_{1 / 2}^{+}} \leq C \sigma .
$$


Let $x^{0} \in B_{R / 2}^{+} \backslash B_{1 / 2}^{+}$. Consider the following two cases:

Rescale

CASE 1. $\left|x^{0}\right| \leq 16 x_{n}^{0}$. Then, denoting $\rho:=\left|x^{0}\right| / 32$, we get $B_{2 \rho}\left(x^{0}\right) \subset B_{R}^{+}$.

$$
W(y):=\frac{1}{2 \rho} w\left(x^{0}+2 \rho y\right)
$$

for $y \in B_{1}(0)=: B_{1}$. Then $W \in C^{2, \alpha}\left(B_{1}\right)$. For $y \in B_{1}$ and $x=x^{0}+2 \rho y \in$ $B_{2 \rho}\left(x^{0}\right)$, we have $D w(x)=D W(y)$, and thus

$$
\begin{aligned}
0= & \operatorname{div}_{x}(\mathcal{M}(x, w(x), D w(x)))+g\left(x^{\prime}, v_{0}(x)+w(x)\right)\left(\frac{1}{q_{0}^{-}-q_{0}^{+}}+w_{x_{n}}(x)\right) \\
= & \frac{1}{\rho} \operatorname{div}_{y}\left(\mathcal{M}\left(x^{0}+2 \rho y, 2 \rho W(y), D W(y)\right)\right) \\
& +g\left(x^{0}+2 \rho y, v_{0}\left(x^{0}+2 \rho y\right)+2 \rho W(y)\right)\left(\frac{1}{q_{0}^{-}-q_{0}^{+}}+W_{y_{n}}(y)\right) .
\end{aligned}
$$

Thus, defining in $B_{1} \times \mathbb{R} \times \mathbb{R}^{n}$,

$$
\begin{aligned}
& A(y, z, P)=\mathcal{M}\left(x^{0}+2 \rho y, 2 \rho z, P\right), \\
& B(y, z, P)=g\left(x^{0}+2 \rho y, v_{0}\left(x^{0}+2 \rho y\right)+2 \rho z\right)\left(\frac{1}{q_{0}^{-}-q_{0}^{+}}+p_{n}\right),
\end{aligned}
$$

we see that $W$ satisfies

$$
\operatorname{div}(A(y, W, D W))+B(y, W, D W)=0 \quad \text { in } \quad B_{1} .
$$

Note that, since $\rho=\left|x^{0}\right| / 32 \geq 1 / 64$, we have

$$
\frac{1}{4} \leq 31 \rho \leq\left|x^{0}+\rho y\right| \leq 33 \rho \quad \text { for } y \in B_{1} .
$$

Also, by Proposition 4.2, (4.32), and (4.34),

$$
\|W\|_{L^{\infty}\left(B_{1}\right)} \leq \frac{C \sigma}{\rho^{n-1}} .
$$

Note that, since $\rho=\left|x_{0}\right| / 32 \geq 1 / 64$, then, for sufficiently small $\sigma$,

$$
\frac{1}{1+\left|\left(x_{0}+2 \rho y\right)+2 \rho z e_{n}\right|} \leq \frac{C}{\rho} \text { for any } y \in B_{1} \text { and }|z| \leq\|W\|_{L^{\infty}\left(B_{1}\right)} .
$$

Now, by Proposition 4.1 with the choice of $\varepsilon=\varepsilon_{0}$ and by (3.4), the functions in (4.33) satisfy the conditions of Theorem A.1 (i) in the ball $B_{1}$ with the constants $\lambda, \Lambda, M$, and $M_{1}=\|W\|_{0,0, L^{\infty}\left(B_{1}\right)}$ depending only on the data, and $\left\|A_{x}(\cdot, \cdot, 0)\right\|_{0, \alpha, B_{1} \times \mathbb{R}} \leq \frac{C \sigma}{1+\rho^{n}}, \quad\left\|\left(1+P^{2}\right)^{-1} B\right\|_{0, \alpha, B_{1} \times\left[-M_{1}, M_{1}\right] \times \mathbb{R}^{n}} \leq \frac{C \sigma}{1+\rho^{n+1}}$. 
Thus, by Theorem A.1 (i) in Appendix and (4.35) and using $\rho \geq 1 / 64$, $\|W\|_{2, \alpha, B_{1 / 2}} \leq C \sigma / \rho^{n-1}$. Rescaling back, we get

$$
\frac{1}{\rho}[w]_{0,0, B_{\rho}\left(x^{0}\right)}+[w]_{1,0, B_{\rho}\left(x^{0}\right)}+\rho[w]_{2,0, B_{\rho}\left(x^{0}\right)}+\rho^{1+\alpha}[w]_{2, \alpha, B_{\rho}\left(x^{0}\right)} \leq \frac{C \sigma}{\rho^{n-1}} .
$$

Note that, for any $x \in B_{2 \rho}\left(x^{0}\right)$, there holds $1 / 128 \leq \rho / \delta_{x} \leq 1$. Thus, multiplying the last estimate by $\rho^{n-1}$, we obtain

$$
\|w\|_{2, \alpha, B_{\rho}\left(x^{0}\right)}^{(n-2)} \leq C \sigma .
$$

CASE 2. $\left|x^{0}\right|>16 x_{n}^{0}$. Let $z=\left(x^{\prime}, 0\right) \in \partial B_{R}^{+} \cap\left\{x_{n}=0\right\}$. Then $|z| \geq$ $\left|x^{0}\right|-x_{n}^{0} \geq 15\left|x^{0}\right| / 16$. Let $\rho=\left|x^{0}\right| / 8$. Then $x^{0} \in B_{\rho}^{+}(z)$. Define $W(y)$ for $y \in B_{1}^{+}$by (4.32). Then $W \in C^{2, \alpha}\left(B_{1}^{+}\right)$and satisfies

$$
\begin{aligned}
& \operatorname{div}(A(y, W, D W))+B(y, W, D W)=0 \quad \text { in } B_{1}^{+}, \\
& A^{n}(y, W, D W)=0 \quad \text { on } \Gamma_{0}:=\partial B_{1}^{+} \cap\left\{x_{n}=0\right\},
\end{aligned}
$$

where $A(y, z, P)$ and $B(y, z, P)$ are defined by (4.33) in $B_{1}^{+}$. Note also that (4.34) and (4.35) hold in $B_{1}^{+}$. Then, by Proposition 4.1, we use (4.36) to see that the functions in (4.33) satisfy the conditions of Theorem A.1 in $B_{1}$ with the constants $\lambda, \Lambda, M$, and $M_{1}=\|W\|_{L^{\infty}\left(B_{1}\right)}$ depending only on the data. Moreover, using the fact that $\left|x^{\prime}\right| \geq|x| / 2$ for $x \in B_{\rho}^{+}(z)$ in (4.7) and the other estimates of Proposition 4.1, we get

$$
\|A(\cdot, \cdot, 0)\|_{1, \alpha, B_{1}^{+} \times \mathbb{R}} \leq \frac{C \sigma}{1+\rho^{n}} .
$$

By (3.4), $\left\|\left(1+|P|^{2}\right)^{-1} B\right\|_{0, \alpha, B_{1}^{+} \times\left[-M_{1}, M_{1}\right] \times \mathbb{R}^{n}} \leq C \sigma /\left(1+\rho^{n+1}\right)$. Thus, by Theorem A.1 (ii), $\|W\|_{2, \alpha, B_{1 / 2}} \leq C \sigma / \rho^{n-1}$. Rescaling back, we get

$$
\frac{1}{\rho}[w]_{0,0, B_{\rho}^{+}(z)}+[w]_{1,0, B_{\rho}^{+}(z)}+\rho[w]_{2,0, B_{\rho}^{+}(z)}+\rho^{1+\alpha}[w]_{2, \alpha, B_{\rho}^{+}(z)} \leq \frac{C \sigma}{\rho^{n-1}} .
$$

Multiplying this estimate by $\rho^{n-1}$ and using that $1 / 128 \leq \rho / \delta_{y} \leq 1$ for every $y \in B_{2 \rho}^{+}(z)$, we get

$$
\|w\|_{2, \alpha, B_{\rho}^{+}(z)}^{(n-2)} \leq C \sigma .
$$

Estimates (4.31), (4.37), and (4.38) imply (4.29): Indeed, it only remains to estimate

$$
\delta_{x, y}^{n-1+\alpha} \frac{|D w(x)-D w(y)|}{|x-y|^{\alpha}}
$$


for $x, y \in B_{R / 2}^{+}$in the case $x>2,|x| \geq|y|$, and $|x-y|>|x| / 32$. Then $|x-y|>\delta_{x} / 64$. As (4.31), (4.37), and (4.38) imply $|D w(z)| \leq C \sigma / \delta_{z}^{n-1}$ for any $z \in B_{R / 2}^{+}$, we get

$$
\delta_{x, y}^{n-1+\alpha} \frac{|D w(x)-D w(y)|}{|x-y|^{\alpha}} \leq C \sigma \delta_{y}^{n-1+\alpha} \frac{\frac{1}{\delta_{x}^{n-1}}+\frac{1}{\delta_{y}^{n-1}}}{\delta_{x}^{\alpha}} \leq C \sigma .
$$

The uniqueness follows from the comparison principle, Theorem B.1 (i).

THEOREM 4.1. There exist $\sigma>0$ and $C$ depending only on the data such that, if $\sigma \leq \sigma_{0}$, there exists a unique solution $w \in C^{2, \alpha}\left(\overline{\mathbb{R}_{+}^{n}}\right)$ of the problem:

$$
\begin{array}{ll}
\operatorname{div}(\mathcal{N}(x, w, D w))=0 & \text { in } \mathbb{R}_{+}^{n}, \\
\mathcal{N}^{n}(x, w, D w)=0 & \text { on }\left\{x_{n}=0\right\}, \\
\|w\|_{0,0, \mathbb{R}_{+}^{n}}^{(n-2)}<\infty, &
\end{array}
$$

satisfying

$$
\|w\|_{2, \alpha, \mathbb{R}_{+}^{n}}^{(n-2)} \leq C \sigma .
$$

Proof. Fix a sequence $R_{j} \rightarrow \infty$ as $j \rightarrow \infty$. Let

$$
w_{R_{j}} \in C\left(\overline{B_{R_{j}}^{+}}\right) \cap C^{2, \alpha}\left(\overline{B_{R_{j}}^{+}} \backslash\left(\partial B_{R_{j}} \cap\left\{x_{n}=0\right\}\right)\right)
$$

be the solution of (4.19) with $R=R_{j}$, constructed in Proposition 4.3. By (4.29), a subsequence of $u_{R_{j}}$ converges in $C^{2, \alpha / 2}\left(\overline{B_{10}^{+}}\right)$. A further subsequence converges in $C^{2, \alpha / 2}\left(\overline{B_{20}^{+}}\right)$, etc. By the diagonal procedure, we extract a sequence $w_{R_{j_{k}}}$ which converges in $C^{2, \alpha / 2}$ on every compact subset of $\overline{\mathbb{R}_{+}^{n}}$. The limit $w$ is thus a solution of (4.39). By (4.29), the limit $w$ satisfies (4.40) with the same constant $C$ as in (4.29).

The uniqueness follows from the comparison principle, Theorem B.1 (ii).

Corollary 4.1. Let $\sigma_{0}$ and $C$ be as in Theorem 4.1, and $\sigma \leq \sigma_{0}$. Then there exists a unique solution of problem (3.24)-(3.25) satisfying (3.19).

This is because $v(x)$ is a solution of problem (3.24)-(3.25) satisfying (3.19) if and only if $w:=v-v_{0}$ is a solution of (4.39) satisfying (4.40).

Corollary 4.1 and Proposition 3.1 imply Theorem 2.1.

REMARK 4.1. If, instead of (2.16), we assume only that $\varphi^{-}$satisfies (2.22), then we obtain a solution of Problem A, which belongs to $C^{1, \alpha}$ for $\alpha \in(0,1)$. Precisely, if we fix any $\alpha \in(0,1)$, then the existence part of Theorem 2.1 holds with estimates (2.19) and (2.21) replaced by

$$
\left\|\varphi-\varphi_{0}^{+}\right\|_{1, \alpha, \Omega^{+}}^{(n-2)} \leq C_{1} \sigma
$$


and

$$
\|f\|_{1, \alpha, \mathbb{R}^{n-1}}^{(n-2)} \leq C_{2} \sigma \text {. }
$$

For the proof, we first assume $\varphi^{-} \in C^{2}$ satisfying (2.22) and then follow the same scheme as above. For the general $\varphi^{-} \in C^{1,1}$ satisfying (2.22), we approximate $\varphi^{-}$by $\varphi_{k}^{-} \in C^{2}$ with the same estimate (2.22) and then send to a limit in a subsequence of solutions by using estimates (4.41) for $\varphi_{k}$ and (4.42) for $f_{\varphi_{k}}$.

\section{5. - Stability of free boundaries}

In this section we prove Theorem 2.2. For the supersonic perturbations $\varphi^{-}$and $\hat{\varphi}^{-}$in $\Omega_{1}$ satisfying (1.1) and (2.16), we define their extensions (still denoted) $\varphi^{-}$and $\hat{\varphi}^{-}$to the whole space as in Section 3.1, and consider the corresponding functions $g$ and $\hat{g}$ defined by (3.3), and $F$ and $\hat{F}$ defined by (3.5) for $\varphi^{-}$and $\hat{\varphi}^{-}$, respectively. Furthermore, we consider the solutions $\varphi$ and $\hat{\varphi}$ of Problem A for $\varphi^{-}$and $\hat{\varphi}^{-}$whose existence is provided by Theorem 2.1 , the functions

$$
u(x)=\varphi^{-}(x)-\varphi(x) \quad \text { in } \Omega^{+}(\varphi), \quad \hat{u}(x)=\hat{\varphi}^{-}(x)-\hat{\varphi}(x) \quad \text { in } \Omega^{+}(\hat{\varphi}),
$$

and their hodograph transform images $v, \hat{v} \in C^{2, \alpha}\left(\overline{\mathbb{R}_{+}^{n}}\right)$ defined by (3.16). Our goal is to prove that there exists a function $\Psi$ with the properties described in Theorem 2.2 such that, for any $\varphi_{1}^{-}$and $\varphi_{2}^{-}$as above,

$$
\|v-\hat{v}\|_{2, \alpha, \mathbb{R}_{+}^{n}}^{(n-2)} \leq \Psi\left(\left\|\varphi^{-}-\hat{\varphi}^{-}\right\|_{2, \alpha, \Omega_{1}}^{(n-1)}\right)
$$

if $\sigma>0$ is sufficiently small. Since $f\left(x^{\prime}\right)=v\left(x^{\prime}, 0\right)$ and $\hat{f}\left(x^{\prime}\right)=\hat{v}\left(x^{\prime}, 0\right)$, estimate (5.1) implies (2.23), thus Theorem 2.2.

If a function $\Psi$ described above does not exist, then there exist $\varphi_{k}^{-}$and $\hat{\varphi}_{k}^{-}$, for $k=1, \cdots$, satisfying (1.1) and such that

$$
\begin{aligned}
& \varphi_{k}^{-} \text {satisfy (2.16) with } \sigma \leq \sigma_{0}, \\
& \left\|\varphi_{k}^{-}-\hat{\varphi}_{k}^{-}\right\|_{2, \alpha, \Omega_{1}}^{(n-1)} \leq \frac{1}{k}, \\
& \left\|v_{k}-\hat{v}_{k}\right\|_{2, \alpha, \mathbb{R}_{+}^{n}}^{(n-2)} \geq \varepsilon>0 .
\end{aligned}
$$

In order to derive a contradiction, we notice the following fact.

Lemma 5.1. Let $\kappa>0$. A set $\mathcal{K}_{M}:=\left\{v \in C^{2, \alpha}\left(\overline{\mathbb{R}_{+}^{n}}\right):\|v\|_{2, \alpha, \mathbb{R}_{+}^{n}}^{(\kappa)} \leq M\right\}$ is compact in the space $C^{2, \beta}\left(\overline{\mathbb{R}_{+}^{n}}\right)$ with the norm $\|\cdot\|_{2, \beta, \mathbb{R}_{+}^{n}}$ for $0<\beta<\alpha$, where $\|\cdot\|_{2, \beta, \mathbb{R}_{+}^{n}}$ is the non-weighted Hölder norm. 
ProOF. Let $v_{j} \in \mathcal{K}_{M}$ for $j=1,2, \ldots$ By a standard argument, we can extract a subsequence (still denoted) $v_{j}$, which converges in $C^{2, \beta}$ on every compact subset of $\overline{\mathbb{R}_{+}^{n}}$ to the limit $v$. Then $\|v\|_{2, \alpha, \mathbb{R}_{+}^{n}}^{(\kappa)} \leq M$. It remains to show that $\left\|v_{j}-v\right\|_{2, \beta, \mathbb{R}_{+}^{n}} \rightarrow 0$ as $j \rightarrow \infty$.

Fix $0<\varepsilon<1$. Then $\left\|v_{j}\right\|_{2, \alpha, \mathbb{R}_{+}^{n} \backslash B_{1 / \varepsilon}(0)} \leq M \varepsilon^{\kappa}$, and the same estimate holds for $v$. Also, there exists $j_{0}$ such that, for $j>j_{0},\left\|v_{j}-v\right\|_{2, \beta, \mathbb{R}_{+}^{n} \cap B_{2 / \varepsilon}(0)} \leq \varepsilon^{\kappa}$. Then, for $j>j_{0}$, we have $\left\|v_{j}-v\right\|_{2, \beta, \mathbb{R}_{+}^{n}} \leq C \varepsilon^{k}$, and the assertion is proved.

Denote

$$
w_{k}(x)=v_{k}(x)-v_{0}(x), \quad \hat{w}_{k}(x)=\hat{v}_{k}(x)-v_{0}(x) .
$$

By Theorem 4.1, both $w_{k}$ and $\hat{w}_{k}$ satisfy (4.40). From (5.2),

$$
\left\|w_{k}-\hat{w}_{k}\right\|_{2, \alpha, \mathbb{R}_{+}^{n}}^{(n-2)} \geq \varepsilon>0 .
$$

Denote by $A_{k}(x, P), B_{k}\left(x^{\prime}, z, P\right)$, and $\mathcal{N}_{k}(x, z, P)$ the functions (3.11), (3.23), and (4.3) corresponding to $\varphi_{k}^{-}$for $k=1,2$. Similarly, let $\hat{A}_{k}(x, P), \hat{B}_{k}\left(x^{\prime}, z, P\right)$, and $\hat{\mathcal{N}}_{k}(x, z, P)$ correspond to $\hat{\varphi}_{k}^{-}$. Each $\mathcal{N}_{k}$ and $w_{k}$ satisfy (4.17)-(4.18). The same is true for $\hat{\mathcal{N}}_{k}$ and $\hat{w}_{k}$.

From (5.2), (4.40) (applied to $w_{k}$ and $\hat{w}_{k}$ ), and Lemma 5.1, by selecting a subsequence (kept the same notation), we see that

$$
\begin{array}{ll}
\varphi_{k}^{-} \rightarrow \varphi^{-}, \quad \hat{\varphi}_{k}^{-} \rightarrow \varphi^{-} & \text {in } C^{2, \alpha / 2}\left(\overline{\Omega_{1}}\right), \\
w_{k} \rightarrow w, \quad \hat{w}_{k} \rightarrow \hat{w} & \text { in } C^{2, \alpha / 2}\left(\overline{\mathbb{R}_{+}^{n}}\right) .
\end{array}
$$

Then $\varphi^{-} \in C^{2, \alpha}\left(\overline{\Omega_{1}}\right)$ satisfies (2.16) with $\sigma \leq \sigma_{0}$, and $w$ and $\hat{w}$ satisfy (4.40). Also, both $w$ and $\hat{w}$ satisfy (4.17)-(4.18), where $\mathcal{N}$ is defined by the limiting function $\varphi^{-}$through the expressions (3.11), (3.23), and (4.3). Then, by Theorem B.1 (ii), $w=\hat{w}$.

On the other hand, by (5.3),

$$
\|w-\hat{w}\|_{2, \alpha, \mathbb{R}_{+}^{n}}^{(n-2)} \geq \varepsilon>0 .
$$

This contradiction leads to (5.1), and thus Theorem 2.2.

REMARK 5.1. If, instead of (2.16), we assume that $\varphi^{-}$satisfies (2.24), then we get a stronger version of Theorem 2.2. Namely, assuming that smooth supersonic solutions $\varphi^{-}(x)$ and $\hat{\varphi}^{-}(x)$ of (1.1) satisfy (2.24) with sufficiently small $\sigma$, we conclude that the unique solutions $\varphi(x)$ and $\hat{\varphi}(x)$ of Problem A for $\varphi^{-}(x)$ and $\hat{\varphi}^{-}(x)$, respectively, satisfy

$$
\left\|f_{\varphi}-f_{\hat{\varphi}}\right\|_{2, \alpha, \mathbb{R}^{n-1}}^{(n-2)} \leq C\left\|\varphi^{-}-\hat{\varphi}^{-}\right\|_{3, \alpha, \Omega_{1}}^{(n-1)}
$$


where $f_{\varphi}\left(x^{\prime}\right)$ and $f_{\hat{\varphi}}\left(x^{\prime}\right)$ are the free boundary functions (2.20) of $\varphi(x)$ and $\hat{\varphi}(x)$, respectively. In fact, in this case, problem (4.17)-(4.18) can be solved by using the implicit function theorem as follows. Denote by $C^{m, \alpha,(\kappa)}(\bar{\Omega})$ the set $\left\{u \in C^{m, \alpha}(\bar{\Omega}):\|u\|_{m, \alpha, \bar{\Omega}}^{(\kappa)}<\infty\right\}$ with $m$ a nonnegative integer, $\alpha \in(0,1)$, and $\kappa>0$. Consider the map $\Phi$, which assigns to $\left(\varphi^{-}, w\right)$ the left-hand sides of (4.17)-(4.18), where $\mathcal{N}$ is defined by $\varphi^{-}$through the expressions (3.11), (3.23), and (4.3). It is easy to see from Proposition 4.1 that $\Phi$ is a $C^{1}$ map from $C^{3, \alpha,(n-1)}\left(\Omega_{1}\right) \times C^{2, \alpha,(n-2)}\left(\overline{\mathbb{R}_{+}^{n}}\right)$ to $C^{0, \alpha,(n+1)}\left(\overline{\mathbb{R}_{+}^{n}}\right) \times C^{1, \alpha,(n)}\left(\mathbb{R}^{n-1}\right)$, for which the higher regularity $\varphi^{-} \in C^{3, \alpha,(n-1)}\left(\overline{\mathbb{R}_{+}^{n}}\right)$ is required. Also, from the definitions, $\Phi\left(\varphi_{0}^{-}, 0\right)=(0,0)$. In order to apply the implicit function theorem, it suffices to show that the partial Fréchet derivative $\Phi_{w}\left(\varphi_{0}^{-}, 0\right)$ is invertible, that is, to show that the conormal problem for the linear elliptic equation:

$$
\sum_{i, j=1}^{n} B_{0 p_{j}}^{i}\left(D v_{0}\right) W_{x_{i} x_{j}}=\psi \text { in } \mathbb{R}_{+}^{n}, \quad \sum_{j=1}^{n} B_{0 p_{j}}^{n}\left(D v_{0}\right) W_{x_{j}}=h \text { on } \partial \mathbb{R}_{+}^{n}
$$

has a unique solution $W \in C^{2, \alpha,(n-2)}\left(\overline{\mathbb{R}_{+}^{n}}\right)$ for any $(\psi, h) \in C^{0, \alpha,(n+1)}\left(\overline{\mathbb{R}_{+}^{n}}\right) \times$ $C^{1, \alpha,(n)}\left(\mathbb{R}^{n-1}\right)$, and $W$ satisfies

$$
\|W\|_{2, \alpha, \mathbb{R}_{+}^{n}}^{(n-2)} \leq C\left(\|\psi\|_{0, \alpha, \mathbb{R}_{+}^{n}}^{(n+1)}+\|h\|_{1, \alpha, \mathbb{R}^{n-1}}^{(n)}\right) .
$$

To construct such a solution, we apply the argument of Section 4 to our linear problem. Now, from the implicit function theorem, we get the existence and uniqueness of solutions in Problem A with small $\sigma$, and stability in the form (5.7).

\section{6. - Multidimensional transonic shocks near spheres}

In this section we study perturbations of spherical transonic shocks to construct multidimensional transonic shock solutions in unbounded domains. Spherical transonic shock solutions in bounded domains were constructed in [6, Section 7]. We first notice that these solutions also exist in the unbounded domains. Precisely, choosing any

$$
0<R_{1}<R_{0}<\infty
$$

we consider the domain $\Omega=\left\{x \in \mathbb{R}^{n}:|x|>R_{1}\right\}$ and show that there exists a weak solution $\varphi_{0} \in W^{1, \infty}(\Omega)$ of (1.1)-(1.2) in the sense of (2.1) with $\varphi_{0}(x)=w(|x|)$ for some $w: \mathbb{R} \rightarrow \mathbb{R}$ such that $\varphi_{0} \in C^{\infty}\left(\overline{\Omega_{0}^{ \pm}}\right)$and

$$
\Omega_{0}^{-}=\left\{x \in \mathbb{R}^{n}: R_{1}<|x|<R_{0}\right\} \quad \text { and } \quad \Omega_{0}^{+}=\left\{x \in \mathbb{R}^{n}:|x|>R_{0}\right\}
$$


are respectively supersonic and subsonic regions of $\varphi_{0}(x)$, i.e.,

$$
\left|D \varphi_{0}\right|>c_{*} \text { in } \Omega_{0}^{-}, \quad\left|D \varphi_{0}\right|<c_{*} \text { in } \Omega_{0}^{+},
$$

and $\left|D \varphi_{0}\right|$ has a jump across $S_{0}:=\left\{|x|=R_{0}\right\}$.

As showed in [6, Section 7], $w(r)$ should satisfy

$$
\Phi\left(w^{\prime}(r)\right)=\frac{\omega}{r^{n-1}},
$$

where $\Phi(\cdot)$ is defined by (2.5) and $\omega$ is a constant. In order to write the solutions explicitly, we first note from (2.6)-(2.9) that there exist smooth functions

$$
\left.\Phi_{+}^{-1}:\left(0, \Phi\left(c_{*}\right)\right) \rightarrow\left(0, c_{*}\right), \quad \Phi_{-}^{-1}:\left(0, \Phi\left(c_{*}\right)\right) \rightarrow\left(c_{*}, \sqrt{2 /(\gamma-1}\right)\right),
$$

which are the inverse functions of $\Phi(\cdot)$ in the sense that $\Phi\left(\Phi_{ \pm}^{-1}(\tau)\right)=\tau$ for any $\tau \in\left(0, \Phi\left(c_{*}\right)\right)$ such that $\left(\Phi_{+}^{-1}\right)^{\prime}(\tau)>0$ and $\left(\Phi_{-}^{-1}\right)^{\prime}(\tau)<0$ for $\tau \in\left(0, \Phi\left(c_{*}\right)\right)$. Thus, in order to satisfy (6.1) and (6.2), we have to choose $\omega>0$ such that $0<\omega / R_{1}^{n-1}<\Phi\left(c_{*}\right)$, and set

$$
w^{\prime}(r)= \begin{cases}\Phi_{-}^{-1}\left(\frac{\omega}{r^{n-1}}\right) & \text { for } R_{1}<r<R_{0} \\ \Phi_{+}^{-1}\left(\frac{\omega}{r^{n-1}}\right) & \text { for } r>R_{0}\end{cases}
$$

Thus, we obtain a weak solution $\varphi_{0}(x)=w(|x|)$ of (1.1) in $\Omega$, satisfying (6.1), by setting

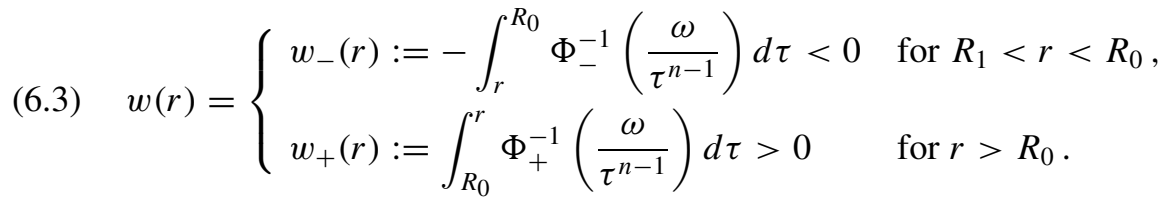

We can express the function $\varphi_{0}(x)$ as

$$
\varphi_{0}(x)=\min \left(\varphi_{0}^{+}(x), \varphi_{0}^{-}(x)\right),
$$

where $\varphi_{0}^{ \pm} \in C^{\infty}(\bar{\Omega})$ are defined as

$$
\varphi_{0}^{ \pm}(x)=w_{ \pm}(|x|) \quad \text { for } R_{1}<r<R_{2} .
$$

The solution $\varphi_{0}(x)$ containing the spherical transonic shock satisfies the entropy condition:

$$
\rho\left(\left|D \varphi_{0}^{-}\right|^{2}\right)<\rho\left(\left|D \varphi_{0}^{+}\right|^{2}\right)
$$

across the transonic shock from the hyperbolic to elliptic phase, which is the direction of fluid motion. 
We now state our results on the existence and stability of multidimensional transonic shocks that are close to the solution $\varphi_{0}(x)$. As in the case of nearplane transonic shocks, we need to specify the supersonic perturbation $\varphi^{-}$only in some neighborhood of the unperturbed shock $S_{0}$, say, in the domain $\Omega_{1}:=$ $\left\{x \in \mathbb{R}^{n}: R_{1}<|x|<R_{0}+1\right\}$.

Problem A'. Given a supersonic solution $\varphi^{-}(x)$ of (1.1) in $\Omega_{1}$, which is a $C^{2, \alpha}$ perturbation of $\varphi_{0}^{-}(x)$ for some $\alpha>0$ :

$$
\left\|\varphi^{-}-\varphi_{0}^{-}\right\|_{C^{2, \alpha}\left(\Omega_{1}\right)} \leq \sigma
$$

with $\sigma>0$ small, find a transonic shock solution $\varphi(x)$ in $\Omega$ such that $\Omega^{-} \subset \Omega_{1}$ and $\varphi(x)=\varphi^{-}(x)$ in $\Omega^{-}$, where $\Omega^{-}$is the supersonic region of $\varphi(x)$, and

$$
\varphi=\varphi^{-}, \quad \partial_{\nu} \varphi=\partial_{\nu} \varphi^{-} \quad \text { on }\left\{|x|=R_{1}\right\} .
$$

THEOREM 6.1. Let $n \geq 3$. There exist positive constants $\sigma_{0}, C_{1}$, and $C_{2}$, depending only on $n, \gamma, \omega$, and $\Omega$, such that, for every $\sigma \leq \sigma_{0}$ and any supersonic solution $\varphi^{-}(x)$ of (1.1) satisfying the conditions stated in Problem $\mathrm{A}^{\prime}$, there exists a unique solution $\varphi(x)$ of Problem $\mathrm{A}^{\prime}$ satisfying

$$
\left\|\varphi-\varphi_{0}^{+}\right\|_{2, \alpha, \Omega^{+}}^{(n-2)} \leq C_{1} \sigma,
$$

and $\Omega^{+}=\left\{|x|>f\left(\frac{x}{|x|}\right)\right\} \cap \Omega$, where $f: S^{n-1} \rightarrow \mathbb{R}$ with

$$
\left\|f-R_{0}\right\|_{2, \alpha, S^{n-1}} \leq C_{2} \sigma .
$$

Moreover, there exists a nonnegative nondecreasing function $\Psi \in C([0, \infty))$ satisfying $\Psi(0)=0$ such that, if $\sigma<\sigma_{0}$ and smooth supersonic solutions $\varphi^{-}(x)$ and $\hat{\varphi}^{-}(x)$ of (1.1) in $\Omega_{1}$ satisfy (6.5), then the unique solutions $\varphi(x)$ and $\hat{\varphi}(x)$ of Problem $\mathrm{A}^{\prime}$ for $\varphi^{-}(x)$ and $\hat{\varphi}^{-}(x)$, respectively, satisfy

$$
\left\|f_{\varphi}-f_{\hat{\varphi}}\right\|_{2, \alpha, S^{n-1}} \leq \Psi\left(\left\|\varphi^{-}-\hat{\varphi}^{-}\right\|_{2, \alpha, \Omega_{1}}\right),
$$

where $f_{\varphi}\left(x^{\prime}\right)$ and $f_{\hat{\varphi}}\left(x^{\prime}\right)$ are the free boundary functions (2.20) of $\varphi(x)$ and $\hat{\varphi}(x)$, respectively.

The proof of this theorem is based on a version of hodograph transform adapted to the geometry of the near-spherical shock: instead of the $x_{n}$-direction in Section 3, we make the transform with respect to the radial direction in the polar coordinates. Now we sketch the proof, focusing on the differences from the case of near-plane transonic shocks.

We first introduce some notations in the sketch of proof below. We use the polar coordinates $(r, \theta) \in \mathbb{R}^{+} \times S^{n-1}$, defined by $r=|x|$ and $\theta=x /|x| \in S^{n-1}$ for $x \in \mathbb{R}^{n} \backslash\{0\}$. 
A function $f(x)$ on $\mathbb{R}^{n} \backslash\{0\}$ is expressed in the polar coordinates as a function $\tilde{f}(r, \theta)$ defined by

$$
\tilde{f}(r, \theta)=f\left(r \frac{\theta}{|\theta|}\right) \quad \text { for }(r, \theta) \in \mathbb{R} \times \mathbb{R}^{n} \backslash\{0\} .
$$

Then

$$
f(x)=\tilde{f}\left(|x|, \frac{x}{|x|}\right) .
$$

Below write $f(r, \theta)$ for $\tilde{f}(r, \theta)$.

STEP 1. Similar to Section 3.1, we extend $\varphi^{-}$from $\Omega_{1}=\left\{x \in \mathbb{R}^{n}: R_{1}<\right.$ $\left.|x|<R_{0}+1\right\}$ to $\mathbb{R}^{n}$ so that the extension (still denoted) $\varphi^{-}$is in $C^{2, \alpha}\left(\mathbb{R}^{n}\right)$ and satisfies (3.1) and

$$
\operatorname{supp}\left(\varphi^{-}-\varphi_{0}^{-}\right) \subset\left\{x \in \mathbb{R}^{n}:|x|<R_{0}+2\right\} .
$$

We also consider the function $g$ defined by (3.3). Moreover, we modify the definition (3.5) as follows: we express the function $g(\cdot)$ in the polar coordinates $(r, \theta) \in(0, \infty) \times S^{n-1}$ and define

$$
F(r, \theta)=\int_{R_{0}}^{r} g(s, \theta) s^{n-1} d s \text { in } \mathbb{R}^{n} .
$$

Then $g$ and $F$ satisfy

$$
\begin{aligned}
& g \in C^{\alpha}\left(\mathbb{R}^{n}\right), \quad\|g\|_{0, \alpha, \mathbb{R}^{n}}^{(n+1)} \leq C \sigma, \quad \operatorname{supp}(g) \cap \Omega \subset \mathbb{R}^{n} \backslash\left\{R_{1}<|x|<R_{0}+1\right\} \\
& F, \partial_{r} F \in C^{\alpha}\left(\mathbb{R}^{n}\right), \quad\|F\|_{0, \alpha, \Omega_{1}} \leq C \sigma, \quad\left\|\partial_{r} F\right\|_{0, \alpha, \mathbb{R}^{n}} \leq C \sigma \\
& \operatorname{supp}(F) \cap \Omega \subset \mathbb{R}^{n} \backslash\left\{R_{1}<|x|<R_{0}+1\right\}
\end{aligned}
$$

Now Problem $\mathrm{A}^{\prime}$ can be stated as the following free boundary problem.

Problem $\mathrm{B}^{\prime}$. Find $\varphi \in C(\Omega)$ and $f \in C^{2, \alpha}\left(S^{n-1}\right)$ such that

(i) In $\Omega$,

$$
\varphi \leq \varphi^{-}
$$

(ii) $\varphi \in C^{2, \alpha}\left(\overline{\Omega^{+}}\right)$with $\Omega^{+}=\left\{\varphi<\varphi^{-}\right\}$, the noncoincidence set;

(iii) $\varphi$ is a solution of (1.1) in $\Omega^{+}$;

(iv) The free boundary $S=\partial \Omega^{+}$is given by the equation $r=f(\theta)$ for $\theta \in S^{n-1}$ in the polar coordinates so that $\Omega^{+}=\{r>f(\theta)\}$;

(v) The free boundary condition (2.3) holds on $S$.

Then a solution of Problem $\mathrm{B}^{\prime}$ satisfying (6.7) and (6.8) is a solution of Problem $\mathrm{A}^{\prime}$, provided that $\sigma$ is sufficiently small. 
STEP 2. Now we perform the partial hodograph transform with respect to the radial direction. Let $\varphi$ be a solution of Problem $\mathrm{A}^{\prime}$ satisfying (6.7)-(6.8).

Define a function $u$ in $\Omega^{+}$by

$$
u(x)=\varphi^{-}(x)-\varphi(x) .
$$

Then (6.7) and the extension properties of $\varphi^{-}$imply

$$
\left\|u-\left(\varphi_{0}^{-}-\varphi_{0}^{+}\right)\right\|_{2, \alpha, \Omega^{+}}^{(n-2)} \leq C \sigma .
$$

In particular, from (6.2) and (6.4), if $\sigma$ is sufficiently small, then

$$
0<\frac{1}{2}\left(\Phi_{-}^{-1}\left(\frac{\omega}{R_{1}^{n-1}}\right)-\Phi_{+}^{-1}\left(\frac{\omega}{R_{1}^{n-1}}\right)\right) \leq \partial_{r} u(x) \leq \sqrt{2 /(\gamma-1)} \text { for any } x \in \Omega^{+} \text {. }
$$

From the definition, $u(x)$ is a solution of (3.10)-(3.11). Now we note that, since $0 \notin \Omega$, we have $\operatorname{div}\left(x /|x|^{n}\right)=0$ in $\Omega$, and thus

$\operatorname{div}\left(\frac{x}{|x|^{n}} F(x)\right)=\frac{x \cdot D F(x)}{|x|^{n}}+F(x) \operatorname{div}\left(\frac{x}{|x|^{n}}\right)=\frac{1}{|x|^{n-1}} \partial_{r} F(x)=g(x)$ in $\Omega$,

where $F$ is defined by (6.13). Now, since $F$ vanishes on $S$, we can rewrite (3.10) as the following conormal boundary value problem:

$$
\begin{array}{ll}
\operatorname{div}\left(A(x, D u)+\frac{x}{|x|^{n}} F(x)\right)=0 & \text { in } \Omega^{+}, \\
\left(A(x, D u)+\frac{x}{|x|^{n}} F(x)\right) \cdot v=0 & \text { on } S .
\end{array}
$$

The weak form of this problem is

$$
\int_{\Omega^{+}}\left(A(x, D u)+\frac{x}{|x|^{n}} F\right) \cdot D \eta d x=0 \quad \text { for any } \eta \in C_{0}^{1}\left(\mathbb{R}^{n}\right) .
$$

We use the notations (6.10)-(6.11) to write (6.19) in the polar coordinates as

$$
\int_{\{u>0\}}\left(A\left(r \theta, \theta \partial_{r} u+\frac{1}{r} D_{\theta} u\right)+\frac{\theta}{r^{n-1}} F(r \theta)\right) \cdot\left(\theta \partial_{r} \eta+\frac{1}{r} D_{\theta} \eta\right) r^{n-1} d r d \theta=0
$$

for any $\eta \in C_{0}^{1}\left(\mathbb{R}^{n}\right)$.

Now, since $u_{r} \geq c>0$ by (6.16), we perform the hodograph transform, i.e., define a map $\Phi: \overline{\Omega^{+}} \rightarrow \mathbb{R}^{n}$ by

$$
(r, \theta) \rightarrow(\rho, \theta)=(u(r, \theta)+1, \theta) .
$$


Since $\Omega^{+}:=\{u>0\}=\{r>f(\theta)\}$ where $f(\theta)$ satisfies (6.8), and $u_{r} \geq c>0$ holds, we have

$$
\Phi\left(\Omega^{+}\right)=\mathbb{R}^{n} \backslash B_{1}(0), \quad \Phi(S)=\partial B_{1}(0),
$$

i.e., the free boundary is mapped to the fixed sphere $\partial B_{1}(0)$. By (6.16), there exists a function $v \in C^{2, \alpha}\left(\mathbb{R}^{n} \backslash B_{1}(0)\right)$ such that, for $(r, \theta)$ satisfying $r \theta \in \overline{\Omega^{+}}$ and $\rho \geq 0$,

$$
u(r, \theta)=\rho-1 \quad \text { if and only if } v(\rho, \theta)=r .
$$

Thus

$$
\Phi^{-1}(\rho, \theta)=(v(\rho, \theta), \theta) .
$$

Differentiating the identity $u(v(\rho, \theta), \theta)=\rho-1$, which holds for any $(r, \theta)$ satisfying $r \theta \in \overline{\Omega^{+}}$, we find

$$
v_{\rho}>0 \quad \text { in } \mathbb{R}^{n} \backslash \overline{B_{1}(0)}
$$

and

$$
D_{\theta} u=-\frac{1}{v_{\rho}} D_{\theta} v, \quad u_{r}=\frac{1}{v_{\rho}},
$$

where the left-hand and right-hand sides are taken at the points $(r, \theta)$ and $\Phi(r, \theta)$, respectively. In particular, (6.16) implies

$$
0<c \leq v_{\rho}(y) \leq C \text { for any }|y|>1 .
$$

From this and (6.7), we get

$$
\left\|v-v_{0}\right\|_{2, \alpha,\{|y|>1\}}^{(n-2)} \leq C \sigma,
$$

where $v_{0}(y) \equiv v_{0}(|y|)$ is the result of the hodograph transform in the radial direction, applied to the function $u_{0}(x) \equiv u_{0}(r):=\varphi_{0}^{-}(r)-\varphi_{0}^{+}(r)$, i.e., $v_{0}(\rho)$ is the unique solution $r$ of the equation $u_{0}(r)=\rho-1$. Note that, from (6.2) and (6.4), $u_{0}(x)$ satisfies (6.16) and thus $v_{0}(y)$ satisfies

$$
0<c \leq \partial_{\rho} v_{0}(y)=\left|D v_{0}(y)\right| \leq C \quad \text { for any } \quad|y|>1 .
$$

Now, we rewrite (6.20) in terms of $v(\rho, \theta)$ by repeating the corresponding calculations from Section 3.3 to arrive at the following equation for $v(\rho, \theta)$ :

$$
\begin{aligned}
& \int_{\{\rho>1\}}\left(A\left(v \theta, \frac{1}{v_{\rho}}\left(\theta-\frac{1}{v} D_{\theta} v\right)\right)+\frac{\theta}{v^{n-1}} F(v \theta)\right) \\
& \cdot\left(\theta \partial_{\rho} \psi+\frac{1}{v}\left(v_{\rho} D_{\theta} \psi-\psi_{\rho} D_{\theta} v\right)\right) v^{n-1} d \rho d \theta=0
\end{aligned}
$$


for any $\psi \in C_{0}^{1}\left(\mathbb{R}^{n}\right)$. Finally, we change the polar coordinates $(\rho, \theta)$ to the Euclidean coordinates $y$ using the formulas (6.10)-(6.11) to get the following equation for $v(y)$ :

$$
\begin{gathered}
\int_{\{|y|>1\}}\left(A\left(\frac{v}{|y|} y, \frac{|y|}{D v \cdot y}\left(\frac{y}{|y|}-\frac{|y|}{v}\left(I-\frac{y \otimes y}{|y|^{2}}\right) D v\right)\right)+\frac{y}{|y| v^{n-1}} F\left(\frac{v}{|y|} y\right)\right) \\
\cdot\left(\frac{y \otimes y}{|y|^{2}} D \psi+\frac{y \cdot D v}{v}\left(I-\frac{y \otimes y}{|y|^{2}}\right) D \psi\right. \\
\left.-\frac{y \cdot D \psi}{v}\left(I-\frac{y \otimes y}{|y|^{2}}\right) D v\right)\left(\frac{v}{|y|}\right)^{n-1} d y=0
\end{gathered}
$$

for any $\psi \in C_{0}^{1}\left(\mathbb{R}^{n}\right)$, where $D=D_{y}, I$ is the $n \times n$ identity matrix, and $y \otimes y$ is the matrix $\left[y_{i} y_{j}\right]_{i, j=1}^{n}$. Clearly, (6.27) is a weak form of the conormal boundary value problem

$$
\begin{array}{ll}
\operatorname{div}(B(y, v, D v)+\tilde{F}(y, v))=0 & \text { in } \mathbb{R}^{n} \backslash \overline{B_{1}(0)}, \\
(B(y, v, D v)+\tilde{F}(y, v)) \cdot \frac{y}{|y|}=0 & \text { on } \partial B_{1}(0),
\end{array}
$$

where

$$
\begin{aligned}
& B(y, z, P)=\left(\frac{y \cdot A(Q)}{|y|^{2}} y+\frac{y \cdot P}{z} A(Q)-\frac{P \cdot A(Q)}{z} y\right)\left(\frac{z}{|y|}\right)^{n-1}, \\
& Q=\left(\frac{z}{|y|} y, \frac{|y|}{P \cdot y}\left(\frac{y}{|y|}-\frac{|y|}{z}\left(I-\frac{y \otimes y}{|y|^{2}}\right) P\right)\right) \\
& \tilde{F}(y, z)=\frac{y}{|y|^{n}} F\left(\frac{z}{|y|} y\right)
\end{aligned}
$$

with $y, P \in \mathbb{R}^{n}$, and $z>0$.

From (6.25), it follows that, if $\sigma$ is small and a solution $v(y)$ of (6.28)(6.29) satisfies (6.24), then (6.23) also holds, and thus we can apply the inverse hodograph transform to $v(y)$ and obtain a solution $u(x)$ of (3.10)-(3.11) satisfying (6.15). Thus, in order to prove the existence and uniqueness part of Theorem 6.1, it suffices to prove the existence and uniqueness of the solution $v(y)$ of (6.28)-(6.29) satisfying (6.24).

STEP 3. Now we show that (6.28)-(6.29) is uniformly elliptic on the function $v(y)$ satisfying (6.24). By a direct (but lengthy) computation, one can check that

$$
\sum_{i, j=1}^{n} B_{p_{j}}^{i}(y, z, P) \xi_{i} \xi_{j}=\left(\frac{z}{|y|}\right)^{n-1} \sum_{i, j=1}^{n} A_{p_{j}}^{i}(Q) \zeta_{i} \zeta_{j},
$$


where $Q$ is defined by (6.31) and

$$
\zeta_{i}=\frac{|y|}{P \cdot y}\left(y_{i} \frac{\xi \cdot y}{|y|^{2}}+\frac{P \cdot y}{z}\left(\xi_{i}-y_{i} \frac{\xi \cdot y}{|y|^{2}}\right)-\frac{\xi \cdot y}{z}\left(p_{i}-y_{i} \frac{P \cdot y}{|y|^{2}}\right)\right) .
$$

We first show that equation (6.28) is uniformly elliptic on $v_{0}(y)$. Indeed, from its definition $v_{0}(1)=R_{0}$, thus, by (6.25),

$$
0<c \leq \frac{v_{0}(y)}{|y|} \leq C \quad \text { for } \quad|y|>1 .
$$

Also, $v_{0}(y)$ is radial: $v_{0}(y) \equiv v_{0}(|y|)$, and thus $D v_{0}(y)=\left|D v_{0}(y)\right| \frac{y}{|y|}$. Therefore, for $(y, z, P)=\left(y, v_{0}(y), D v_{0}(y)\right)$ with $|y|>1$,

$$
\zeta=\frac{1}{\left|D v_{0}(y)\right|}\left(\frac{y \otimes y}{|y|^{2}}+\left|D v_{0}(y)\right| \frac{|y|}{v_{0}(y)}\left(I-\frac{y \otimes y}{|y|^{2}}\right)\right) \xi=: H \xi .
$$

From (6.25) and (6.34), the matrix $H$ satisfies

$$
c I \leq H \leq C I
$$

for some constants $C>c>0$ depending only on $n$ and the constants in (6.25) and (6.34). We also note that, from the definitions, $v_{0}(y) \equiv v_{0}(|y|)$ satisfies

$$
v_{0}^{\prime}(\rho)=\frac{1}{\left(w^{-}\right)^{\prime}(r)-\left(w^{+}\right)^{\prime}(r)},
$$

where $w(r)$ is from (6.4), and $r$ is the unique solution of $w^{-}(r)-w^{+}(r)=\rho-1$. Thus

$$
D v_{0}(y)=\frac{1}{\left(w^{-}\right)^{\prime}(|y|)-\left(w^{+}\right)^{\prime}(|y|)} \frac{y}{|y|} .
$$

Then, for $(y, z, P)=\left(y, v_{0}(y), D v_{0}(y)\right)$ with $|y|>1, Q$ defined by (6.31) takes the form

$$
\begin{aligned}
Q & =\left(\frac{v_{0}(y)}{|y|} y, \frac{1}{\left|D v_{0}(y)\right|} \frac{y}{|y|}\right)=\left(\frac{v_{0}(y)}{|y|} y,\left(\left(w^{-}\right)^{\prime}(r)-\left(w^{+}\right)^{\prime}(r)\right) \frac{y}{|y|}\right) \\
& =\left(x, D \varphi_{0}^{-}(x)-D \varphi_{0}^{+}(x)\right),
\end{aligned}
$$

where $x=r \frac{y}{|y|} \in \Omega^{+}$. Since (1.1) is uniformly elliptic on $\varphi_{0}^{+}(x)$ for $x \in$ $\mathbb{R}^{n} \backslash B_{R_{1}}(0)$, it follows from (3.1) and (3.11) that

$$
\lambda I \leq\left[A_{q_{j}}^{i}(Q)\right] \leq \Lambda I,
$$

where $\Lambda>\lambda>0$ depend only on the data. From (6.33)-(6.36), we conclude that (6.28)-(6.29) is uniformly elliptic on $v_{0}(y)$ and thus on the function $v$ satisfying (6.24) if $\sigma$ is small. 
STEP 4. The rest of the argument follows closely the argument of Sections 4 and 5 .

First, we modify $B(y, z, P)$ away from a neighborhood of $\left(y, v_{0}(y), D v_{0}(y)\right)$ to obtain a uniformly elliptic equation globally, and also restate problem (6.28)(6.29) in the terms of the function

$$
w(y)=v(y)-v_{0}(y) .
$$

In order to do that, we note that $v_{0}(y)$ is a solution of the problem of form (6.28)-(6.29) with $B_{0}(y, z, P)$ which corresponds to the supersonic solution $\varphi_{0}^{-}(y)$, i.e., $B_{0}(y, z, P)$ is defined by (3.23) with $A_{0}(x, P)$, defined by (3.11) with $\varphi_{0}^{-}(x)$ instead of $\varphi^{-}(x)$. Then $\tilde{F}_{0} \equiv 0$. Now, similar to (4.3), we define

$$
\begin{aligned}
\mathcal{N}(y, z, P)= & D_{P} B_{0}\left(y, v_{0}(y), D v_{0}(y)\right) \cdot P+\eta(|P|)\left(B(X(y, z, P))-L_{0}(P)\right) \\
& +\tilde{F}\left(y, v_{0}(y)+z\right),
\end{aligned}
$$

where

$$
\begin{aligned}
& X(y, z, P):=\left(y, v_{0}(y)+z, D v_{0}(y)+P\right), \\
& L_{0}(P):=B_{0}\left(y, v_{0}(y), D v_{0}(y)\right)+D_{P} B_{0}\left(y, v_{0}(y), D v_{0}(y)\right) \cdot P,
\end{aligned}
$$

and $\eta$ is defined in Section 4. Then, for sufficiently small $\sigma$, if $v(y)$ is a solution of (6.28)-(6.29) satisfying (6.24), then $w(y)$ is a solution of the conormal problem

$$
\begin{array}{ll}
\operatorname{div}(\mathcal{N}(y, w, D w))=0 & \text { in } \mathbb{R}^{n} \backslash \overline{B_{1}(0)}, \\
\mathcal{N}(y, w, D w) \cdot \frac{y}{|y|}=0 & \text { on } \partial B_{1}(0)
\end{array}
$$

and

$$
\|w\|_{2, \alpha, \Omega^{+}}^{(n-2)} \leq C \sigma .
$$

In order to find such a solution, we first show that $\mathcal{N}$ is uniformly elliptic and satisfies the estimates of Proposition 4.1. Then we solve problem (6.38)(6.39) in the bounded domains $B_{R}(0) \backslash \overline{B_{1}(0)}$ prescribing $w=0$ on $\partial B_{R}(0)$. For such problems, we show the estimates of the solution $w=w_{R}$ independent of $R$ : First we show the weighted $L^{\infty}$ estimate, which is obtained by the argument of Proposition 4.2, in which we use the comparison function

$$
V(x)=L\left(|x|^{2-n}-\mu|x|^{2-n+1 / 2}\right)
$$

with singularity at the origin. The argument is slightly simplified in the present case since the boundary $\partial B_{1}(0)$ is compact. Then we obtain the weighted $C^{2, \alpha}$ estimates by following Case 1 of the proof of Proposition 4.3 and using the compactness of the boundary. After obtaining the existence, uniqueness, and uniform estimates of solutions in the bounded domains $B_{R}(0) \backslash \overline{B_{1}(0)}$, we send $R$ to infinity and obtain a solution of (6.38)-(6.39) in the unbounded domain $\mathbb{R}^{n} \backslash \overline{B_{1}(0)}$ as in Theorem 4.1. The uniqueness in $\mathbb{R}^{n} \backslash \overline{B_{1}(0)}$ follows from the comparison principle similar to Theorem B.1 (ii). This establishes the existence and uniqueness part of Theorem 6.1. The stability follows by repeating the argument of Section 5. 


\section{Appendix A. Local estimates of solutions of the conormal problems}

The following facts follow by combining some standard results on elliptic equations with our new formulations.

THEOREM A.1.

(i) Let $B_{r}:=B_{r}(0) \subset \mathbb{R}^{n}$. Let $u \in C^{2}\left(B_{1}\right)$ be a solution of the equation

$$
\operatorname{div}(A(x, u, D u))+B(x, u, D u)=0 \quad \text { in } B_{1} .
$$

Assume also that

$$
\|u\|_{L_{\left(B_{1}\right)}} \leq M_{1} .
$$

Denote $\mathcal{D}:=B_{1} \times\left[-M_{1}, M_{1}\right] \times \mathbb{R}^{n}$ and $\mathcal{D}_{x, z}:=B_{1} \times\left[-M_{1}, M_{1}\right]$. Assume that $A(x, z, P)$ and $B(x, z, P)$ satisfy

$$
\begin{aligned}
& \|A(\cdot, \cdot, P)\|_{0, \alpha, \mathcal{D}_{x, z}} \leq M(1+|P|) \quad \text { for any } P \in \mathbb{R}^{n}, \\
& \left\|\left(D_{P} A, A_{z}, D_{x} A, D_{P x}^{2} A\right)\right\|_{0, \alpha, \mathcal{D}} \leq M, \\
& \left\|\left(1+|P|^{2}\right)^{-1} B\right\|_{0, \alpha, \mathcal{D}} \leq M \text { for any } P \in \mathbb{R}^{n} .
\end{aligned}
$$

Assume that $A(x, z, P)$ is elliptic, i.e., there exist $\Lambda \geq \lambda>0$ such that

$$
\lambda|\xi|^{2} \leq \sum_{i, j=1}^{n} A_{p_{j}}^{i}(x, z, P) \xi_{i} \xi_{j} \leq \Lambda|\xi|^{2} \quad \text { for any } \xi \in \mathbb{R}^{n},(x, z, P) \in \mathcal{D} .
$$

Then $u \in C^{2, \alpha}\left(B_{1 / 2}\right)$ and there exists $C$ depending only on $n, \lambda, \Lambda, M, M_{1}$, and $\alpha$ such that

$$
\|u\|_{2, \alpha, B_{1 / 2}} \leq C\left(\|u\|_{L^{\infty}\left(B_{1}\right)}+\left\|D_{x} A(\cdot, \cdot, 0)\right\|_{0, \alpha, \mathcal{D}_{x, z}}+\left\|\left(1+|P|^{2}\right)^{-1} B\right\|_{0, \alpha, \mathcal{D}}\right) .
$$

(ii) Let $B_{r}^{+}=B_{r}(0) \cap\left\{x_{n}>0\right\} \subset \mathbb{R}^{n}$. Let $u \in C^{2}\left(B_{1}^{+}\right)$be a solution of the conormal problem:

$$
\begin{aligned}
& \operatorname{div}(A(x, u, D u))+B(x, u, D u)=0 \quad \text { in } B_{1}^{+}, \\
& A^{n}(x, u, D u)=0 \quad \text { on } \Gamma_{0}:=\partial B_{1}^{+} \cap\left\{x_{n}=0\right\} .
\end{aligned}
$$

Let $u(x)$ and $A(x, z, P)$ satisfy all the assumptions of (i) above in the domains $B_{1}^{+}, \mathcal{D}^{+}:=B_{1}^{+} \times\left[-M_{1}, M_{1}\right] \times \mathbb{R}^{n}$, and $\mathcal{D}_{x, z}^{+}:=B_{1}^{+} \times\left[-M_{1}, M_{1}\right]$. In addition, assume that the function $\left(x^{\prime}, z\right) \rightarrow A\left(\left(x^{\prime}, 0\right), z, 0\right)$ satisfies

$$
\left\|(1+|P|)^{-1} A\right\|_{1, \alpha, \mathcal{D}^{\prime}} \leq M,
$$

where $\mathcal{D}^{\prime}:=\left(B_{1} \cap\left\{x_{n}=0\right\}\right) \times\left[-M_{1}, M_{1}\right] \times \mathbb{R}^{n}$. Then $u \in C^{2, \alpha}\left(\overline{B_{1 / 2}^{+}}\right)$and there exists $C$ depending only on $n, \lambda, \Lambda, M, M_{1}$, and $\alpha$ such that

$$
\begin{aligned}
\|u\|_{2, \alpha, B_{1 / 2}^{+}} \leq & C\left(\|u\|_{L^{\infty}\left(B_{1}^{+}\right)}+\left\|D_{x} A(\cdot, \cdot, 0)\right\|_{0, \alpha, \mathcal{D}_{x, z}}+\|A(\cdot, \cdot, 0)\|_{1, \alpha, \mathcal{D}_{x, z}^{\prime}}\right. \\
& \left.+\left\|\left(1+|P|^{2}\right)^{-1} B\right\|_{0, \alpha, \mathcal{D}^{+}}\right),
\end{aligned}
$$

where $\mathcal{D}_{x, z}^{\prime}:=\left(B_{1} \cap\left\{x_{n}=0\right\}\right) \times\left[-M_{1}, M_{1}\right]$. 
Proof. We sketch only the proof of assertion (ii) since the proof of (i) is similar. The constant $C$ below depends only on $n, \lambda, \Lambda, M, M_{1}$, and $\alpha$, and may be different at each occurrence.

Using condition (A.1) and the assumptions on $A(x, z, P)$ and $B(x, z, P)$, we can apply a local version of the estimates in [23, Section 5] to obtain

$$
\|D u\|_{0, \alpha, B_{7 / 8}^{+}} \leq C \text {. }
$$

Now we rewrite the equation in (A.4) in the nondivergence form:

$$
\sum_{i, j=1}^{n} A_{p_{j}}^{i}(x, u, D u) u_{x_{i} x_{j}}+\sum_{i=1}^{n}\left(A_{z}^{i}(x, u, D u) u_{x_{i}}+A_{x_{i}}^{i}(x, u, D u)\right)+B(x, u, D u)=0 .
$$

Using (A.1)-(A.3), (A.5), and (A.6), we can apply the local estimates from the proof of [23, Theorem 2] to obtain

$$
\|u\|_{2, \alpha, B_{3 / 4}^{+}} \leq C .
$$

Now, in $B_{5 / 8}^{+}$, we can rewrite the conormal boundary value problem as a linear problem:

$$
\begin{aligned}
& \sum_{i, j=1}^{n} a_{i j}(x) u_{x_{i} x_{j}}+\sum_{i=1}^{n} b_{i}(x) u_{x_{i}}=f(x) \quad \text { in } B_{1}^{+}, \\
& \sum_{i=1}^{n} c_{i}(x) u_{x_{i}}=g(x) \quad \text { on } \quad \Gamma_{0}:=\partial B_{1}^{+} \cap\left\{x_{n}=0\right\},
\end{aligned}
$$

where

$$
\begin{aligned}
a_{i j}(x) & =A_{p_{j}}^{i}(x, u(x), D u(x)), \\
b_{i}(x) & =A_{z}^{i}(x, u(x), D u(x))+\sum_{j=1}^{n} \int_{0}^{1} A_{x_{j} p_{i}}^{j}(x, u(x), t D u(x)) d t, \\
f(x) & =-\sum_{i=1}^{n} A_{x_{i}}^{i}(x, u(x), 0)-B(x, u(x), D u(x)), \\
c_{i}(x) & =\int_{0}^{1} A_{p_{i}}^{n}(x, u(x), t D u(x)) d t, \quad g(x)=-A^{n}(x, u(x), 0) .
\end{aligned}
$$

From the ellipticity of $A$, condition (A.2), and estimate (A.7), we have

$$
\begin{aligned}
& \sum_{i, j}\left\|a_{i j}\right\|_{1, \alpha, B_{5 / 8}^{+}}+\sum_{i}\left\|b_{i}\right\|_{0, \alpha, B_{5 / 8}^{+}}+\sum_{i}\left\|c_{i}\right\|_{1, \alpha, B_{5 / 8}^{+}} \leq C, \\
& c_{n}\left(x^{\prime}, 0\right) \geq \lambda, \\
& \|f\|_{0, \alpha, B_{5 / 8}^{+}} \leq C\left(\left\|D_{x} A(\cdot, \cdot, 0)\right\|_{0, \alpha, \mathcal{D}_{x, z}^{+}}+\|B\|_{0, \alpha, \mathcal{D}^{+} \cap\left\{|P| \leq\|u\|_{C^{1}\left(B_{3 / 4}^{+}\right)}\right)}\right) \\
& \quad \leq C\left(\left\|D_{x} A(\cdot, \cdot, 0)\right\|_{0, \alpha, \mathcal{D}_{x, z}^{+}}+\left\|\left(1+|P|^{2}\right)^{-1} B\right\|_{0, \alpha, \mathcal{D}^{+}}\right), \\
& \|g\|_{1, \alpha, B_{5 / 8} \cap\left\{x_{n}=0\right\}} \leq C\|A(\cdot, \cdot, 0)\|_{1, \alpha, \mathcal{D}_{x, z}^{\prime}} \cdot
\end{aligned}
$$

Now assertion (ii) follows from the standard linear estimates, see e.g. [16, Lemma 6.29]. 


\section{Appendix B. Comparison principles for the conormal problems} lems.

We now show the following comparison principles for the conormal probTHEOREM B.1.

(i) Suppose $u_{1}, u_{2} \in C\left(\overline{B_{R}^{+}}\right) \cap C^{1}\left(B_{R}^{+}\right)$, and

$$
\begin{aligned}
& \operatorname{div}\left(A\left(x, u_{1}, D u_{1}\right)\right) \leq \operatorname{div}\left(A\left(x, u_{2}, D u_{2}\right)\right) \quad \text { in } B_{R}^{+}, \\
& A^{n}\left(x, u_{1}, D u_{1}\right) \leq A^{n}\left(x, u_{2}, D u_{2}\right) \quad \text { on } \Gamma_{0}:=\partial B_{R}^{+} \cap\left\{x_{n}=0\right\} \\
& u_{1} \geq u_{2} \text { on } \Gamma_{1}:=\partial B_{R}^{+} \cap\left\{x_{n}>0\right\}
\end{aligned}
$$

in the weak sense, i.e., for any nonnegative $\varphi \in C^{1}\left(\overline{B_{R}^{+}}\right)$satisfying $\varphi=0$ on $\Gamma_{1}$,

$$
\int_{B_{R}^{+}} A\left(x, u_{1}, D u_{1}\right) \cdot D \varphi(x) d x \geq \int_{B_{R}^{+}} A\left(x, u_{2}, D u_{2}\right) \cdot D \varphi(x) d x .
$$

Assume that

$$
\left.A, A_{z}, D_{P} A \in C^{1} \overline{\left(B_{R}^{+}\right.} \times \mathbb{R} \times \mathbb{R}^{n}\right)
$$

with $\left\|\left(A_{z}, D_{P} A\right)\right\|_{L^{\infty}\left(B_{R}^{+} \times \mathbb{R} \times \mathbb{R}^{n}\right)} \leq M<\infty$ and that the operator $A$ is elliptic, i.e., (A.3) holds for all $(x, z, P) \in \overline{B_{R}^{+}} \times \mathbb{R} \times \mathbb{R}^{n}$. Then

$$
u_{1} \geq u_{2} \quad \text { in } B_{R}^{+}
$$

(ii) Let $n \geq 3$. Suppose $u_{1}, u_{2} \in C^{1, \alpha}\left(\overline{\mathbb{R}_{+}^{n}}\right)$ with $\left\|u_{k}\right\|_{1, \alpha, \mathbb{R}_{+}^{n}}^{(n-2)} \leq M_{1}<\infty$ for $k=1,2$, and

$$
\begin{aligned}
& \operatorname{div}\left(A\left(x, u_{1}, D u_{1}\right)\right) \leq \operatorname{div}\left(A\left(x, u_{2}, D u_{2}\right)\right) \quad \text { in } \mathbb{R}_{+}^{n}, \\
& A^{n}\left(x, u_{1}, D u_{1}\right) \leq A^{n}\left(x, u_{2}, D u_{2}\right) \quad \text { on } \Gamma_{0}:=\left\{x_{n}=0\right\}
\end{aligned}
$$

in the weak sense, i.e., for any nonnegative $\varphi \in C_{c}^{1}\left(\mathbb{R}^{n}\right)$.

$$
\int_{\mathbb{R}_{+}^{n}} A\left(x, u_{1}, D u_{1}\right) \cdot D \varphi(x) d x \geq \int_{\mathbb{R}_{+}^{n}} A\left(x, u_{2}, D u_{2}\right) \cdot D \varphi(x) d x .
$$

Assume that

$$
A, A_{z}, D_{P} A \in C^{1}\left(\overline{\mathbb{R}_{+}^{n}} \times \mathbb{R} \times \mathbb{R}^{n}\right)
$$

with $\left|D_{P} A(x, z, P)\right|+\left|\left(1+|x|^{m}\right) A_{z}(x, z, P)\right| \leq M<\infty$ for some $m>n / 2$ and for any $(x, z, P) \in \overline{\mathbb{R}_{+}^{n}} \times \mathbb{R} \times \mathbb{R}^{n}$ with $|z|+|P| \leq M_{1}$. Assume that the operator $A$ is elliptic, i.e., (A.3) holds for any $(x, z, P) \in \overline{\mathbb{R}_{+}^{n}} \times \mathbb{R} \times \mathbb{R}^{n}$. Then

$$
u_{1} \geq u_{2} \quad \text { in } \mathbb{R}_{+}^{n}
$$


Proof. (i). We follow and modify the proof of [16, Theorem 10.7 (ii)]. Let $w=u_{2}-u_{1}$. Then $w \leq 0$ on $\Gamma_{0}$ and, from (B.1),

$$
\int_{B_{R}^{+}}\left(\sum_{i, j=1}^{n} a_{i j}(x) w_{x_{j}} \varphi_{x_{i}}+\sum_{i=1}^{n} b_{i}(x) w \varphi_{x_{i}}\right) d x \leq 0
$$

for any nonnegative $\varphi \in C^{1}\left(\overline{B_{R}^{+}}\right)$satisfying $\varphi=0$ on $\Gamma_{1}$, where

$$
\begin{aligned}
a_{i j}(x) & =\int_{0}^{1} A_{p_{j}}^{i}\left(x,(1-t) u_{1}(x)+t u_{2}(x),(1-t) D u_{1}(x)+t D u_{2}(x)\right) d t \\
b_{i}(x) & =\int_{0}^{1} A_{z}^{i}\left(x,(1-t) u_{1}(x)+t u_{2}(x),(1-t) D u_{1}(x)+t D u_{2}(x)\right) d t
\end{aligned}
$$

Note that $a_{i j}, b_{i} \in C\left(B_{R}^{+}\right)$with $\left\|\left(a_{i j}, b_{i}\right)\right\|_{L^{\infty}\left(B_{R}^{+}\right)} \leq M$, by the assumptions.

We need to prove that $w \leq 0$ in $B_{R}^{+}$. By approximation, (B.2) holds for any nonnegative $\varphi \in W^{1,2}\left(B_{R}^{+}\right)$satisfying $\varphi=0$ on $\Gamma_{1}$. Thus, for any $\varepsilon>0$, we can substitute $\varphi=w^{+} /\left(w^{+}+\varepsilon\right)$ into (B.2) with $w^{+}=\max (w, 0)$. Then, repeating the calculations in [16, page 270], we obtain

$$
\int_{B_{R}^{+}}\left|D \log \left(1+w^{+} / \varepsilon\right)\right|^{2} d x \leq C(\Lambda, \lambda, M, R) .
$$

Since $\log \left(1+w^{+} / \varepsilon\right)=0$ on $\Gamma_{1}$, it follows from the Poincaré inequality that

$$
\int_{B_{R}^{+}}\left|\log \left(1+w^{+} / \varepsilon\right)\right|^{2} d x \leq C(\Lambda, \lambda, M, R) .
$$

Letting $\varepsilon \rightarrow 0$, we conclude $w^{+} \equiv 0$ in $B_{R}^{+}$, i.e., $w \leq 0$.

(ii) Similar to the case of the half-ball, we consider the function $w=u_{2}-u_{1}$. It satisfies

$$
\|w\|_{1, \alpha, \mathbb{R}_{+}^{n}}^{(n-2)} \leq 2 M_{1} .
$$

Also, for any nonnegative $\varphi \in C_{c}^{1}\left(\mathbb{R}^{n}\right)$,

$$
\int_{\mathbb{R}_{+}^{n}}\left(\sum_{i, j=1}^{n} a_{i j}(x) w_{x_{j}} \varphi_{x_{i}}+\sum_{i=1}^{n} b_{i}(x) w \varphi_{x_{i}}\right) d x \leq 0,
$$

where $a_{i j}$ and $b_{i}$ are defined by (B.3). Note that, by the assumptions, $a_{i j}, b_{i} \in$ $C\left(\overline{\mathbb{R}^{n}}\right)$ with

$$
\left|a_{i j}(x)\right|+\left|\left(1+|x|^{m}\right) b_{i}(x)\right| \leq M \quad \text { for any } x \in \mathbb{R}_{+}^{n} .
$$


By approximation, (B.4) holds for any nonnegative $\varphi \in W^{1,2}\left(\mathbb{R}_{+}^{n}\right)$ satisfying $\varphi=0$ a.e. on $\mathbb{R}_{+}^{n} \backslash B_{R}(0)$ for some $R>0$. Thus, for any $\varepsilon>0$ and $R>0$, we can substitute $\varphi=w^{+} \eta_{R}^{2} /\left(w^{+}+\varepsilon\right)$ into (B.4), where $\eta_{R}(x)=\eta\left(\frac{x}{R}\right)$ with $\eta \in C_{c}^{\infty}\left(\mathbb{R}^{n}\right)$ satisfying

$$
\eta \geq 0 \quad \text { in } \mathbb{R}^{n}, \quad \eta \equiv 1 \quad \text { in } B_{1}(0), \quad \eta \equiv 0 \quad \text { in } \quad \mathbb{R}^{n} \backslash B_{2}(0) .
$$

Substituting $\varphi$ defined above into (B.4), we use the summation convention to get

$$
\begin{aligned}
& \int_{\mathbb{R}_{+}^{n}}\left(a_{i j} w_{x_{i}}^{+} w_{x_{j}}^{+} \frac{\varepsilon \eta_{R}^{2}}{\left(w^{+}+\varepsilon\right)^{2}}+b_{j} w^{+} w_{x_{j}}^{+} \frac{\varepsilon \eta_{R}^{2}}{\left(w^{+}+\varepsilon\right)^{2}}\right. \\
& \left.\quad+2 a_{i j} w_{x_{i}}^{+} \eta_{R}\left(\eta_{R}\right)_{x_{j}} \frac{w^{+}}{w^{+}+\varepsilon}+b_{j} w^{+} \eta_{R}\left(\eta_{R}\right)_{x_{j}} \frac{w^{+}}{w^{+}+\varepsilon}\right) d x \leq 0 .
\end{aligned}
$$

Thus, using the ellipticity, estimate (B.5) with $m>\frac{n}{2}$, and the properties of $\eta_{R}$, we have

$$
\begin{aligned}
& \lambda \int_{B_{R}^{+}}\left|D \log \left(1+w^{+} / \varepsilon\right)\right|^{2} d x \leq \lambda \int_{\mathbb{R}_{+}^{n}} \eta_{R}^{2}\left|D \log \left(1+w^{+} / \varepsilon\right)\right|^{2} d x \\
& =\lambda \int_{\mathbb{R}_{+}^{n}} \eta_{R}^{2} \frac{\left|D w^{+}\right|^{2}}{\left(w^{+}+\varepsilon\right)^{2}} d x \leq \int_{\mathbb{R}_{+}^{n}}\left(a_{i j} w_{x_{i}}^{+} w_{x_{j}}^{+} \frac{\varepsilon \eta_{R}^{2}}{\left(w^{+}+\varepsilon\right)^{2}}\right) d x \\
& \leq C(n, M, \Lambda)\left(\int_{B_{R}^{+}}+\int_{B_{2 R}^{+} \backslash B_{R}^{+}}\right) \frac{1}{1+|x|^{m}} \frac{w^{+}}{w^{+}+\varepsilon}\left|D \log \left(1+w^{+} / \varepsilon\right)\right| d x \\
& \quad+\frac{C(n, M, \Lambda)}{\varepsilon R} \int_{B_{2 R}^{+} \backslash B_{R}^{+}}\left(\frac{w^{+}\left|D w^{+}\right|}{w^{+}+\varepsilon}+\frac{1}{1+|x|^{m}} \frac{\left|w^{+}\right|^{2}}{w^{+}+\varepsilon}\right) d x \\
& \leq C(n, M, \Lambda)\left(\int_{\mathbb{R}^{n}} \frac{1}{1+|x|^{2 m}} d x\right)^{\frac{1}{2}}\left(\int_{B_{R}^{+}}\left|D \log \left(1+w^{+} / \varepsilon\right)\right|^{2} d x\right)^{\frac{1}{2}} \\
& \quad+\frac{C\left(n, M, M_{1}, \Lambda\right)}{\varepsilon^{2}}\left(\frac{R^{n}}{R^{m} R^{n-1} R^{n-2}}+\frac{R^{n}}{R R^{n-2} R^{n-1}}+\frac{R^{n}}{R R^{m} R^{2(n-2)}}\right),
\end{aligned}
$$

where, in the last inequality, we used the following estimates:

$$
|w(x)| \leq \frac{M_{1}}{1+|x|^{n-2}}, \quad|D w(x)| \leq \frac{M_{1}}{1+|x|^{n-1}} .
$$

Since $m>n / 2$, we obtain

$$
\int_{B_{R}^{+}}\left|D \log \left(1+w^{+} / \varepsilon\right)\right|^{2} d x \leq C\left(n, m, M, M_{1}, \Lambda, \lambda\right)\left(1+\varepsilon^{-2} R^{-(n-2)}\right) .
$$


Since $n \geq 3$, then sending $R \rightarrow \infty$ yields

$$
\int_{\mathbb{R}_{+}^{n}}\left|D \log \left(1+w^{+} / \varepsilon\right)\right|^{2} d x \leq C\left(n, m, M, M_{1}, \Lambda, \lambda\right) .
$$

Now we extend $w$ to $\mathbb{R}^{n}$ by the reflection $w\left(x^{\prime},-x_{n}\right):=w\left(x^{\prime}, x_{n}\right)$ for $x_{n}>0$ and continue to denote the extension by $w$. Then $w \in C^{0,1}\left(\mathbb{R}^{n}\right)$, and (B.6) holds in $\mathbb{R}^{n}$ (the estimate of $|D u|$ holds a.e.). Also, from (B.7),

$$
\int_{\mathbb{R}^{n}}\left|D \log \left(1+w^{+} / \varepsilon\right)\right|^{2} d x \leq C\left(n, m, M, M_{1}, \Lambda, \lambda\right) .
$$

Now consider the functions

$$
v(x):=\log \left(1+w^{+} / \varepsilon\right), \quad v_{R}(x):=\eta_{R}(x) \log \left(1+w^{+} / \varepsilon\right) \quad \text { in } \mathbb{R}^{n},
$$

where $R>0$ and $\eta_{R}$ is defined above. Then $v \in C^{0,1}\left(\mathbb{R}^{n}\right)$ and $v_{R} \in C_{c}^{0,1}\left(\mathbb{R}^{n}\right)$. Thus, we use $n \geq 3$ to obtain

$$
\left(\int_{\mathbb{R}^{n}}\left|v_{R}\right|^{\frac{2 n}{n-2}} d x\right)^{\frac{n-2}{n}} \leq C(n) \int_{\mathbb{R}^{n}}\left|D v_{R}\right|^{2} d x .
$$

Since

$$
|v(x)| \leq \frac{1}{\varepsilon} w^{+}(x) \leq \frac{M_{1}}{\varepsilon} \frac{1}{1+|x|^{n-2}}
$$

and $D v \in L^{2}\left(\mathbb{R}^{n}\right)$ by (B.8), we use the similar properties of $\eta_{R}$ as in the estimates above and $n \geq 3$ to see that the left-hand and right-hand sides of (B.9) converge, as $R \rightarrow \infty$, to the left-hand and right-hand sides of the inequality

$$
\left(\int_{\mathbb{R}^{n}}|v|^{\frac{2 n}{n-2}} d x\right)^{\frac{n-2}{n}} \leq C(n) \int_{\mathbb{R}^{n}}|D v|^{2} d x
$$

respectively. Now, by (B.8),

$$
\int_{\mathbb{R}^{n}}\left|\log \left(1+w^{+} / \varepsilon\right)\right|^{\frac{2 n}{n-2}} d x \leq C\left(n, m, M, M_{1}, \Lambda, \lambda\right) .
$$

Since this is true for any $\varepsilon>0$, we conclude that $w^{+} \equiv 0$ in $\mathbb{R}^{n}$, i.e., $w \leq 0$.

\section{REFERENCES}

[1] H. W. Alt - L. A. Caffarelli, Existence and regularity for a minimum problem with free boundary, J. Reine Angew. Math. 325 (1981), 105-144. 
[2] H. W. Alt - L. A. Caffarelli - A. Friedman, A free-boundary problem for quasilinear elliptic equations, Ann. Scuola Norm. Sup. Pisa Cl. Sci. (4) 11 (1984), 1-44.

[3] H. W. Alt - L. A. Caffarelli - A. Friedman, Compressible flows of jets and cavities, J. Differential Equations 56 (1985), 82-141.

[4] L. Bers, Existence and uniqueness of subsonic flows past a given profile, Comm. Pure Appl. Math. 7 (1954), 441-504.

[5] S. Canić - B. L. Keyfitz - G. Lieberman, A proof of existence of perturbed steady transonic shocks via a free boundary problem, Comm. Pure Appl. Math. 53 (2000), 484511.

[6] G.-Q. Chen - M. Feldman, Multidimensional transonic shocks and free boundary problems for nonlinear equations of mixed type, J. Amer. Math. Soc. 16 (2003), 461-494.

[7] G.-Q. Chen - M. Feldman, Steady transonic shocks and free boundary problems in infinite cylinders for the Euler equations, Comm. Pure Appl. Math. 57 (2004), 310-356.

[8] S.-X. CHEN, Existence of stationary supersonic flows past a point body, Arch. Rational Mech. Anal. 156 (2001), 141-181.

[9] S.-X. Chen, Asymptotic behavior of supersonic flow past a convex combined wedge, Chinese Ann. Math. 19B (1998), 255-264.

[10] R. Courant - K. O. Friedrichs, "Supersonic Flow and Shock Waves", Springer-Verlag, New York, 1948.

[11] C. M. Dafermos, "Hyperbolic Conservation Laws in Continuum Physics", SpringerVerlag, Berlin, 2000.

[12] G. Dong, "Nonlinear Partial Differential Equations of Second Order", Transl. Math. Monographs, 95, AMS, Providence, RI, 1991.

[13] L. C. Evans - W. Gangbo, Differential equations methods for the Monge-Kantorovich mass transfer problem, Mem. Amer. Math. Soc. n. 653, 137 (1999).

[14] R. Finn - D. Gilbarg, Asymptotic behavior and uniqueness of plane subsonic flows, Comm. Pure Appl. Math. 10 (1957), 23-63.

[15] R. Finn - D. Gilbarg, Three-dimensional subsonic flows, and asymptotic estimates for elliptic partial differential equations, Acta Math. 98 (1957), 265-296.

[16] D. Gilbarg - N. Trudinger, "Elliptic Partial Differential Equations of Second Order", 2nd Ed., Springer-Verlag, Berlin, 1983.

[17] J. Glimm - A. Majda, "Multidimensional Hyperbolic Problems and Computations", Springer-Verlag, New York, 1991.

[18] C.-H. Gu, A method for solving the supersonic flow past a curved wedge (in Chinese), J. Fudan Univ. Nat. Sci. 7 (1962), 11-14.

[19] D. Kinderlehrer - L. Nirenberg, Regularity in free boundary problems, Ann. Scuola Norm. Sup. Pisa Cl. Sci. (4) 4 (1977), 373-391.

[20] P. D. Lax, "Hyperbolic Systems of Conservation Laws and the Mathematical Theory of Shock Waves", CBMS-RCSM, SIAM, Philiadelphia, 1973.

[21] T.-T. LI, On a free boundary problem, Chinese Ann. Math. 1 (1980), 351-358.

[22] G. Lieberman, Regularity of solutions of nonlinear elliptic boundary value problems, J. Reine Angew. Math. 369 (1986), 1-13.

[23] G. Lieberman, Hölder continuity of the gradient of solutions of uniformly parabolic equations with conormal boundary conditions, Ann. Mat. Pura Appl. (4) 148 (1987), 77-99. 
[24] G. Lieberman, Mixed boundary value problems for elliptic and parabolic differential equations of second order, J. Math. Anal. Appl. n. 2, 113 (1986), 422-440.

[25] G. LieBERMAN - N. TRUDINGER, Nonlinear oblique boundary value problems for nonlinear elliptic equations, Trans. Amer. Math. Soc. 295 (1986), 509-546.

[26] W.-C. LiEn - T.-P. Liu, Nonlinear stability of a self-similar 3-dimensional gas flow, Comm. Math. Phys. 204 (1999), 525-549.

[27] A. MAJDA, "The stability of multidimensional shock fronts", Mem. Amer. Math. Soc. n. 275, 41, AMS, Providence, 1983.

[28] A. Majda, "The existence of multidimensional shock fronts", Mem. Amer. Math. Soc. n. 281, 43, AMS, Providence, 1983.

[29] G. MÉTIVIER, Stability of multi-dimensional weak shocks, Comm. Partial Differential Equations 15 (1990), 983-1028.

[30] C. S. Morawetz, On the non-existence of continuous transonic flows past profiles I-III, Comm. Pure Appl. Math. 9 (1956), 45-68; 10 (1957), 107-131; 11 (1958), 129-144.

[31] O. Oleinik, "Some Asymptotic Problems in the Theory of Partial Differential Equations", Lezioni Lincee. [Lincei Lectures] Cambridge University Press, Cambridge, 1996.

[32] D. G. SchaefFer, Supersonic flow past a nearly straight wedge, Duke Math. J. 43 (1976), 637-670.

[33] M. Shiffman, On the existence of subsonic flows of a compressible fluid, J. Rational Mech. Anal. 1 (1952), 605-652.

[34] Y. Zhang, Steady supersonic flow past an almost straight wedge with large vertex angle, J. Differential Equations 192 (2003), 1-46.

Department of Mathematics Northwestern University Evanston, IL 60208-2730, USA gqchen@math.northwestern.edu

Department of Mathematics University of Wisconsin Madison, WI 53706-1388, USA feldman@math.wisc.edu 